Rincon-Novoa, J., Rojas-Berrio, S. \& Garcia-Peña, B. (2021). Accountability, marketing relacional y grupos de interés en el transporte aéreo colombiano. Contaduría Universidad de Antioquia, 79, 215-251. Doi: https://doi.org/10.17533/ udea.rc.n79a09

\title{
Accountability, marketing relacional y grupos de interés en el transporte aéreo colombiano
}

Jeisson Rincon-Novoa

Universidad Nacional de Colombia

jlrinconn@unal.edu.co

Orcid: 0000-0002-1834-5141

Sandra Rojas-Berrio

Universidad Nacional de Colombia

sprojasb@unal.edu.co

Orcid: 0000-0002-1148-3779

Bibiana Garcia-Peña

Universidad Nacional de Colombia

bpgarciap@unal.edu.co

Orcid: 0000-0002-6290-2152 
Accountability, marketing relacional y grupos de interés en el transporte aéreo colombiano

Resumen: Producto de las relaciones existentes entre las organizaciones con sus grupos de interés o stakeholders, se han desarrollado estrategias de comunicación de sus acciones e iniciativas en aspectos ambientales, económicos y sociales mediante la emisión de informes anuales como parte de su proceso de rendición de cuentas, de manera que sirvan de base para que los stakeholders evalúen la conducta empresarial. Sin embargo, se ha podido evidenciar que la producción de información tanto financiera como no financiera responde a una estrategia de marketing relacional con el fin de conservar solo ciertos grupos de interés dentro de las organizaciones. Asi pues, este trabajo pretende evaluar el comportamiento del accountability como herramienta de marketing relacional dirigido a los grupos de interés dentro del sector de transporte aéreo colombiano, mediante la construcción de una matriz de observación que permita caracterizar dicho comportamiento en el sector.

Palabras clave: Marketing relacional, accountability, reportes de sostenibilidad, grupos de interés, transporte aéreo colombiano.

\section{Accountability, relational marketing and interest groups in Colombian air transport}

Abstract: As a result of existing relationships between organizations and their stakeholders, strategies for communicating their actions and initiatives in environmental, economic, and social aspects have been developed through the publishing of annual reports as part of their accountability process, so that they serve as a basis for stakeholders to evaluate business conduct. However, it has been evidenced that the production of both financial and non-financial information responds to a relationship marketing strategy aimed at retaining only certain stakeholders within the organizations. Thus, this paper aims to evaluate the behavior of accountability as a relationship marketing tool aimed at stakeholders in the Colombian air transport sector, through a systematic literature review and the construction of an observation matrix to characterize such behavior in the sector.

Keywords: Relational Marketing, accountability, sustainability report, stakeholders, Colombian air transport.

\section{Responsabilidade, marketing de relacionamento e grupos de interesse no transporte aéreo colombiano}

Resumo: Produto das relações existentes entre as organizações com seus grupos de interesse ou stakeholders, tem se desenvolvido estratégias de comunicação de suas ações e iniciativas em aspectos ambientais, econômicos e sociais mediante a emissão de relatórios anuais como parte de seu processo de prestação de contas, de maneira que sirvam de base para que os stakeholders avaliem a conduta empresarial. Porém, tem se evidenciado que a produção de informação tanto financeira como não financeira responde a uma estratégia de marketing relacional com o fim de conservar só certos grupos de interesse dentro das organizações. Assim, este trabalho visa avaliar o comportamento do "accountability" como ferramenta de marketing relacional dirigido aos grupos de interesse dentro do setor de transporte aéreo colombiano, através de uma revisão sistemática de literatura e a construção de uma matriz de observação que permita caracterizar tal comportamento no setor.

Palavras chave: Marketing relacional, accountability, relatórios de sustentabilidade, grupos de interesse, transporte aéreo colombiano.

Responsabilisation, marketing relationnel et groupes d'intérêt dans le transport aérien colombien

Résumé: En raison des relations existantes entre les organisations et leurs groupes d'intérêt, des stratégies ont été développées pour communiquer leurs actions et initiatives en matière environnementale, économique et sociale. Ceci est fait par la publication de rapports annuels dans le cadre de leur processus de responsabilisation, afin qu'ils puissent servir de base aux stakeholders pour évaluer la conduite des affaires. Cependant, il est devenu évident que la production d'informations tant financières que non financières répond à une stratégie de marketing relationnel visant à ne retenir que certains groupes d'intérêt au sein des organisations. Ainsi, cet article vise à évaluer le comportement de la "accountability " en tant qu'outil de marketing relationnel destiné aux groupes d'intérêt du secteur du transport aérien colombien, à travers une revue systématique de la littérature et la construction d'une matrice d'observation pour caractériser ce comportement dans le secteur.

Mots clés: marketing relationnel, accountability, rapports de durabilité, groupes d'intérêt, transport aérien colombien. 
Cont. udea (julio-septiembre, pp. 215-251. (C) Universidad de Antioquia-2021.

\title{
Accountability, marketing relacional y grupos de interés en el transporte aéreo colombiano
}

\author{
Jeisson Rincon-Novoa, Sandra Rojas-Berrio, y Bibiana Garcia-Peña \\ https://doi.org/10.17533/udea.rc.n79a09
}

Primera versión recibida en abril de 2021 - Versión aceptada en agosto de 2021

\section{Introducción}

En la actualidad las organizaciones se conciben como sistemas abiertos que interactúan con el entorno en los procesos de creación de valor. Estas interacciones son el resultado de las sinergias entre las diferentes partes interesadas, stakeholders, que mantienen relaciones específicas con la distribución de valor y que tienen la capacidad de afectar y la posibilidad de verse afectado por el logro de los objetivos organizacionales (Freeman, 1984; Freeman, Harrison, Wicks, Parmar, \& de Colle, 2010). En relación con esta interacción, los gestores han desarrollado diversas estrategias de comunicación de sus iniciativas (informes de gestión, memorias de sostenibilidad o reportes integrados) en múltiples ámbitos (ambiental, económico y social), con el fin de divulgarlas de cara a la rendición de cuentas, accountability, como una herramienta de marketing relacional con los stakeholders (Dumitru, Gușe, Feleagă, Mangiuc, \& Feldioreanu, 2015).

De este modo, el desarrollo de reportes anuales de información financiera y no financiera se ha posicionado como un asunto fundamental a incluir dentro del campo de acción del regulador en contextos internacionales. Por ejemplo, en el marco europeo, considerando que es necesario aumentar la transparencia de la información social-medioambiental en relación con el crecimiento sostenible y los intereses de los stakeholders, entre en vigencia la Directiva 2014/95/UE por medio de la cual se plantea la obligatoriedad (esencialmente en las grandes empresas, entidades de interés público) de divulgar este tipo de información y emitir un dictamen de auditoría que proporcione confianza sobre las cifras y afirmaciones de estos informes (Parlamento Europeo \& Consejo de la Unión Europea, 2014). 
Accountability, marketing relacional y grupos de interés en el transporte aéreo colombiano...

Sin embargo, la regulación colombiana ha dejado de lado la noción de obligatoriedad frente a la información de índole no financiera. Esta situación ha generado un abanico de opciones en la estructura de los reportes anuales, a razón de establecer una estrategia para captar recursos económicos o posicionar marcas en el mercado a partir del marketing relacional. De esta manera, la información es vista como un bien privado sujeto a la optimización de oferta y demanda entre los grupos de interés para aquellos que puedan acceder a esta (Deegan, 2014).

Lo anterior permitiría vislumbrar una dinámica de mercado en la producción de la información financiera y no financiera para conservar ciertos grupos de interés dentro de las organizaciones y omitir aspectos relevantes para otros. Frente a ello, algunos autores han manifestado interés por analizar el campo de la divulgación de información no financiera asociado a la presencia en internet y su correlación positiva con el sector económico (Gómez-Meneses \& CatólicoSegura, 2009).

Ahora bien, con base en el análisis de antecedentes,, el enfoque de las investigaciones sobre este tema ha estado distanciado de presentar un análisis sobre el comportamiento de la rendición de cuentas en relación con los intereses y aspectos materiales para cada uno de los stakeholders, el efecto que tienen estos informes en el contexto colombiano para estrechar relaciones entre la organización y ciertos grupos específicos, la influencia que pueden ejercer los stakeholders para sesgar las características de la rendición de cuentas; así como otros planteamientos clave bajo la mirada contractual y de poder, propios del problema de la agencia (Monterrey y Segura, 2008; Ross, 1973; Williamson, 1989; 1996).

La contabilidad en las empresas, bajo la mirada de Sunder (2005), evita que el conflicto entre los integrantes del modelo contractual conlleve a la desintegración de la organización al poner en práctica y hacer cumplir los acuerdos que subyacen en esta. Por su parte, autores como Mattessich hacia finales de los años 90 plantearon una interpretación de lo que se entiende como inicio y fin de la contabilidad. Esto conllevó a que, conforme a lo citado por Rodríguez de Ramírez, la "...principal función...estaría, entonces, en facilitar el funcionamiento fluido de relaciones de accountability entre partes interesadas..." (2012, p. 15); concepto que se ha estudiado ampliamente desde diversas perspectivas (financiera, social, crítica y ciencia política) y es definido como el proceso de rendición de cuentas a los grupos de interés.

En el Siglo XXI las organizaciones han adoptado diversas formas de rendición de cuentas ya sea porque es de obligatorio cumplimiento o por mantener una estrecha relación con sus stakeholders. Dentro de estas se encuentra la divulgación de informes anuales que dan de cuenta tanto resultados financieros propios de la contabilidad, como aspectos de marketing centrados en mitigar 
los impactos negativos y propiciar un desarrollo sustentable. Estos documentos sirven de base para que todos los stakeholders juzguen la conducta empresarial y tomen decisiones a partir de esta (Rodríguez de Ramírez, 2012).

Así pues, este estudio plantea la posibilidad de abordar la información no financiera que hace parte de la rendición de cuentas y sobre esta verificar el grado o no de conversación individual o personalizada con cada uno de los grupos de interés y sus asuntos fundamentales; especialmente, a partir de un análisis empírico y cualitativo dentro del sector de transporte aéreo colombiano.

Esta investigación es relevante para los profesionales e investigadores de las ciencias económicas toda vez que las organizaciones como sistemas abiertos están en constante iteración con el entorno. Así que, evaluar si desde la mirada de la rendición de cuentas se puede gestionar la relación con los grupos de interés, permitirá generar sinergias organizacionales para garantizar que cada uno de estos se sienta identificado y por ende se desarrollen conexiones de largo entre la marca y los usuarios de la información no financiera.

Además, de acuerdo con los resultados de la revisión sistemática de literatura, no se ha evaluado como la accountability es empleada como una estrategia de marketing relacional con los grupos de interés por parte de las organizaciones. Especialmente, en lo que respecta a la divulgación de información no financiera; la cual representa un insumo relevante para el análisis de la conducta empresarial.

En ese sentido, se debe determinar cómo los informes de gestión, memorias de sostenibilidad o reportes integrados, entre otras formas de rendición de cuentas, se encuentran asociados a los planes de marketing a través de una mirada integral hacia la organización. Lo anterior, a partir de la evaluación de los grupos de interés, como lo son; información, accionistas, colaboradores, consumidores, medioambiente, proveedores, gobernanza y sociedad, los cuales se abordan en la herramienta propuesta.

Por otro lado, el sector de transporte aéreo en Colombia se espera sea clave para la recuperación económica del país en época de postpandemia. Esto se debe a la consolidación del sector que se dio a partir de la apertura de nuevas rutas y el aumento en las frecuencias de algunas aerolíneas. Asimismo, cabe destacar la inversión por parte del Gobierno en la infraestructura aeroportuaria, lo que fortaleció la conectividad regional. No obstante, la literatura académica en relación con los procesos de accountability o rendición de cuentas es aún escasa en esta industria.

En ese orden de ideas, es relevante dar una mirada a uno de los sectores que tuvo el mayor crecimiento en los últimos años, el cual a su vez se vio impactado de la misma manera por la pandemia del COVID-19. Así, para noviembre de 2020 se esperaba que el sector aeronáutico sufriera una caída en sus ingresos del $72 \%$ respecto a 2019 y se perdieran alrededor de unos 50.000 empleos (Semana, 
Accountability, marketing relacional y grupos de interés en el transporte aéreo colombiano...

2020), lo que se vio reflejado en una pérdida de US 1.094 millones que presentó Avianca para el año 2020 (Avianca, 2020a; 2020b). Es importante resaltar que el sector está concentrado principalmente en tres operadores, Avianca (53.8\%), Aires LATAM (20\%) y Viva Colombia (15\%) (La República, 2020).

Sin embargo, gracias a la recuperación económica que se ha venido dando progresivamente, Colombia se posiciona como el tercer país en Latinoamérica que más ha recuperado su capacidad aérea internacional después de México y Ecuador, recuperando el $47 \%$ de la conectividad aérea (Procolombia, 2021). Así mismo, entre septiembre de 2020 y enero de 2021 se movilizaron 6.058 .790 pasajeros en Colombia, lo que representa una recuperación importante y progresiva del sector (Aeronáutica Civil, 2021); situación que causa mayor expectativa frente a las estrategias de rendición de cuentas e impactos de la gestión.

Por consiguiente, si la contabilidad media los conflictos entre los grupos de interés y su función principal está en facilitar la relación de rendición de cuentas con estos, se hace relevante evaluar el comportamiento del accountability dentro del contexto empresarial colombiano de transporte aéreo porque si bien se encuentra regulado desde el punto de vista financiero, la divulgación de información no financiera abre la oportunidad de que esta se use como estrategia de comunicación directa (selectiva o no) que mantenga un enfoque hacia determinados grupos de interés.

De esta manera, el objetivo de esta investigación es evaluar el comportamiento del accountability como herramienta de marketing relacional dirigido a los grupos de interés dentro del sector de transporte aéreo colombiano, contrastando las premisas identificadas en el proceso de revisión sistemática de literatura con la práctica empresarial colombiana y caracterizando el comportamiento del sector sobre las estrategias de comunicación relacional de información financiera y no financiera hacia los grupos de interés.

Se presentarán los grupos de interés a los que se dirigen principalmente los mensajes presentes en los informes de sostenibilidad a partir de proponer una matriz de evaluación cualitativa sobre los aspectos materiales que se han investigado académicamente en la literatura. Así mismo, se espera que este trabajo fortalezca el interés de los profesionales e investigadores de las ciencias económicas para analizar la efectividad de la información no financiera en relación con las acciones de marketing que permiten mejorar la comunicación y por ende la lealtad de los grupos de interés con las organizaciones del sector aéreo colombiano que operan en el país.

\section{Metodología}

El paradigma metodológico bajo la cual se guía esta aproximación es de corte interpretativo basado en un razonamiento inductivo, puesto que se partirá desde la perspectiva del comportamiento del accountability en el sector. Los 
métodos por usar son principalmente cualitativos a partir de una estratégica metodológica observacional/archivística y con un horizonte de tiempo longitudinal (De Gortari, 1968; Saunders et al., 2016).

De este modo, inicialmente se abordan los asuntos materiales de los grupos de interés mediante una revisión sistemática de literatura que permita identificar los elementos clave para tener en cuenta en la gestión de la relación. Posteriormente, esta investigación se centra en la construcción, validación con jueces expertos de la matriz de observación y registro, caracterización y evaluación del accountability como herramienta de marketing relacional dirigido a los grupos de interés dentro del sector del transporte aéreo colombiano.

En relación con lo anterior, se llevó a cabo una revisión sistemática siguiendo los protocolos propuestos (Belanche et al., 2020; Beltrán, 2005; Chicaíza-Becerra et al., 2017; Kitchenham et al., 2009, 2010; Paul, 2019; Paul \& Benito, 2018; Pérez-Rave et al., 2012; Rana \& Paul, 2017; Tripathi et al., 2019). El desarrollo de esta fase partió de la elaboración del protocolo de revisión, la definición de la pregunta de investigación, la validación de la ecuación de búsqueda y aplicación en los principales sistemas de indexación y resumen como lo son Elsevier (Scopus) y Thomson Reuters (Web of Sciences). Lo anterior, a partir de dos tópicos generales, el campo del accountability y la "teoría de los stakeholders" o "stakeholders", para luego tamizar los resultados, hacer lectura completa de los documentos seleccionados e identificar las premisas marco de los asuntos materiales para los grupos de interés.

En paralelo, se realizó la revisión y descargue de los informes de sostenibilidad, reportes integrados u otro documento que hiciese alusión a la rendición de cuentas sobre la generación de valor para los grupos de interés. Esto conllevó la búsqueda de los sitios oficiales de las organizaciones que operan dentro del sector de transporte aéreo colombiano y el descargue de las comunicaciones oficiales de sostenibilidad del número de años disponibles sistemáticamente en las unidades de observación.

Seguido a ello, se construyó la matriz de observación y registro como instrumento que permita evaluar la estructura, contenido y concentración de la información divulgada en los procesos de accountability con los diferentes stakeholders. Esta fue revisada, ajustada y retroalimentada por tres jueces expertos; un académico con formación doctoral en contabilidad e investigador de responsabilidad social corporativa y teoría contable, un alto directivo corporativo de planeación y evaluación estratégica en el ámbito local, y un alto directivo de investigaciones sociales y sostenibilidad empresarial en el ámbito nacional.

Así bien, una vez validada la operacionalización de la matriz por medio de jueces expertos, se procedió a realizar el análisis histórico y secuencial de la información no financiera reportada por las organizaciones que componen el sector. Esta evaluación se realizó asignando una calificación de (1) ante la pre- 
Accountability, marketing relacional y grupos de interés en el transporte aéreo colombiano...

sencia de información que mejore la relación con el grupo de interés y (0) ante la ausencia, para finalmente realizar un análisis de concordancia mediante el estadístico W de Kendall.

\section{El accountability y los grupos de interés}

En la literatura se pueden identificar diversas denominaciones y aproximaciones para lo que se conoce como stakeholders o grupos de interés, ya sean colaboradores, clientes, accionistas, medioambiente, gobierno y sociedad (Lee, Seo, \& Sharma, 2013; Luo, Lam, Chau, Shen, \& Wang, 2017; Salmi \& Khan, 2019). De modo que para cualquier investigación se deben determinar sus diferencias al momento de revisar metodologías o modelos (Estándares GRI, Reporte Integrado, ISO 26000, u otros) que suelen agruparlos, a partir de aspectos materiales generales como los asuntos económicos, sociales y ambientales.

Aunado a lo anterior, el incremento de la demanda de información ética y veraz por las partes interesadas (Hinson, 2011; Karaman \& Akman, 2018) y la búsqueda por iniciar procesos de responsabilidad social empresarial (RSE) como estrategia de comunicación en las industrias (Power, 2007; Sweeney \& Coughlan, 2008), ha traído como consecuencia un aumento de la revelación de información no financiera durante las últimas décadas.

En este sentido, algunas investigaciones muestran que comunicar la información de RSE a través de los medios de comercialización traduce esta información en valor para los accionistas por su efecto en los resultados (Kim \& Kim, 2019), y en general una efectiva accion de marketing para conectar los asuntos materiales con los grupos de interes. Incluso, desde el enfoque de los académicos, el uso de las redes sociales como un mecanismo para diseminar la información corporativa ha tenido una baja participación en relación con la comunicación relacionada con los temas de sostenibilidad (Mahmud, Manan, Hashim, Nor, \& Azizan, 2017).

Por otro lado, la literatura muestra que hay una mayor propensión a realizar procesos de RSE en industrias dónde los impactos conllevan efectos negativos sobre la sociedad, evidenciado principalmente en la publicación de informes cada vez más completos en industrias como los juegos de azar (Luo \& Lam, 2016; Luo et al., 2017), bebidas alcohólicas (Babor, Robaina, \& Jernigan, 2015; Jernigan \& Babor, 2015), captación de dinero del público en el sector financiero (Hinson, 2011; Rashid, Abdeljawad, Ngalim, \& Hassan, 2013; Singh \& Agarwal, 2013), modas (Pedersen \& Andersen, 2015), y gas e hidrocarburos (Serry, 2019).

Esta información sirve como una estrategia de marketing que comparte los resultados e impactos obtenidos en determinado periodo de tiempo (Nelson, 2014; Romolini, Fissi, \& Gori, 2014), con el fin de mejorar el relacionamiento, la reputación corporativa con los accionistas, la confianza de los colaboradores 
y la satisfacción a los clientes (Kuo, Kremer, Phuong, \& Hsu, 2016); así como, la relación de largo plazo con todos los grupos de interés (O’Malley, 2014).

A partir del desarrollo de nuevas metodologías de revelación de la información no financiera, diversos grupos de interés como la sociedad, el medio ambiente y los clientes, han tomado importancia dentro de la presentación de la declaración de valor añadido (Dumitru Guşe et al., 2015). Esto implica ir más allá de lo legalmente exigido y responder ante múltiples actores y expectativas, sin descuidar a los accionistas y su rentabilidad, pues esto genera compromisos con las partes interesadas cuyas opiniones pueden variar (Andriof \& McIntosh, 2017).

Así pues, dependiendo del sector y el nivel de interés general, las organizaciones realizan una división de las partes interesadas, ya sea en primarias (quienes no afectan el riesgo para sí mismas) y no primarias (quienes reducen el riesgo para las partes primarias) (Kim \& Kim, 2019), de acuerdo con las matrices de materialidad o el cumplimiento de las expectativas de sus principales stakeholders.

De ahí que existan múltiples enfoques de relacionamiento específico con los grupos de interés, que analizan desde el riesgo de los accionistas a partir de la evaluación del riesgo de la inversión (Batista, 2018; Kim \& Kim, 2019) hasta el riesgo ambiental. En dónde este último normalmente se traduce en acciones dirigidas al green marketing (Gupta \& Agrawal, 2016), que en ocasiones se traslapa como un intento de guardar apariencias, acercarse a un grupo en específico o lograr la legitimación en el mercado (Crocker, 2014).

De esta forma, en gran parte de la academia se ha centrado en establecer una metodología de cálculo mediante pesos relativos en sectores específicos sobre el comportamiento de accountability (Serry, 2019). Se han desarrollado escalas de medición perceptual en economías emergentes para la sostenibilidad (Bachman, Bashyal, \& Baumann, 2012; Luo et al., 2017); la construcción de plantillas de evaluación de implementación y calidad sobre los procesos de rendición de cuentas o accountability (Silvestri, Veltri, Venturelli, \& Petruzzelli, 2017); así como, cuestionarios especializados de evaluación de pares sobre el desempeño de las industrias (Karaman \& Akman, 2018).

Corrientes teóricas alternativas muestran un enfoque hacia el desarrollo de marcos especializados en organizaciones sin ánimo de lucro (Wickham, Lehman, \& French, 2015); así como, la implementación de reportes con enfoque de pensamiento integrado y sistematizado, puesto que se evalúa ampliamente la creación de valor a los stakeholders sin descuidar a los inversores (Batista, 2018). Por otro lado, se ha fortalecido la crítica a los informes fragmentados, al verse como una estrategia externa no relacionada a una de marketing (Hinson, 2011), de tal modo que en ocasiones su divulgación es sesgada e incompleta (Waller \& Lanis, 2009).

Estudios latinoamericanos revelan que en los contextos de la región se presenta información poco detallada sobre la gestión realizada (Da Silva, Seibert, 
Accountability, marketing relacional y grupos de interés en el transporte aéreo colombiano...

Wbatuba, \& Macagnan, 2015); que desde otra perspectiva, se cuestionan las acciones fiscales y éticas sobre la divulgación de este tipo de informes (Groninger, 2010).

En adición, algunas visiones plantean que en el futuro los procesos de rendición de cuentas migrarán a características mutuas, que permitan introducir un nuevo enfoque para aunar esfuerzos sobre divulgación y contraer compromisos conjuntos con los grupos de interés, en favor de los objetivos de desarrollo sostenible (Franklin \& Oehmke, 2019). Esto considerando que la consulta con las partes interesadas se ha llevado a cabo principalmente a través de la creación de consejos consultivos, mientras que la amplitud de la cobertura y la calidad de la retroalimentación mejoran las estructuras para abordar las preocupaciones de las partes interesadas (Irvine \& O’Brien, 2009).

Finalmente, estudios recientes plantean la necesidad de generar conexiones entre las áreas responsables de comunicar los esfuerzos e impactos resultado de la RSE dentro de las organizaciones y la gestión de mercadeo (comercialización, fidelización y otras) (Souza-Monteiro \& Hooker, 2017). De modo que se disemine información transparente, coherente e hilada mediante sus estrategias de marketing y de sostenibilidad (Souza-Monteiro \& Hooker, 2017), buscando así generar valor para todos los grupos de interés (Kim \& Kim, 2019).

\section{Sector de transporte aéreo y factores relevantes a evaluar}

Si bien el sector de transporte aéreo es de especial interés en el contexto empresarial colombiano, la literatura académica en relación con los procesos de accountability o rendición de cuentas es aún escasa en esta industria, ya sea por su característica oligopólica en el mercado o por las condiciones específicas del sector en los diferentes territorios. Algunas investigaciones manifiestan que la adopción ha sido de baja participación entre los actores de la industria aeronáutica comercial (Cowper-Smith \& de Grosbois, 2011).

Por otro lado, algunos estudios de caso muestran una mayor disposición a realizar análisis especializados sobre factores medioambientales, por encima de los enfocados a factores sociales o económicos. Lo anterior se debe principalmente a la búsqueda por reducir las emisiones de carbono, residuos e impacto a las comunidades (Cowper-Smith \& de Grosbois, 2011; Robinson, 2015).

Asimismo, algunas aproximaciones incluyen someramente aspectos de bienestar y compromiso de los empleados, diversidad y equidad social, de la comunidad y la prosperidad económica (Cowper-Smith \& de Grosbois, 2011). Además, entre las diferentes formas de aproximación al comportamiento de accountability se evidencian iniciativas relacionadas con cuestionarios de comparación por pares y expertos miembros del sector aéreo, con el fin de identificar la importancia relativa de los factores relacionados (Karaman \& Akman, 2018). 
Por otra parte, debido a que el enfoque de la investigación busca una mirada integral a los grupos de interés, adicional a lo ya mencionado. Así bien, la literatura plantea diversos factores relevantes a analizar, entre ellos; el diálogo directo con los grupos de interés (Salmi \& Khan, 2019), los aspectos estratégicos organizacionales, el desarrollo de un código de gobierno corporativo y una estructura de gobernanza corporativa (Becerra Velasco, Espinosa Ávila, Guzmán Hurtado, \& Rodríguez Vargas, 2017; Jouini, Ajina, \& Derbali, 2018; Thompson, Alleyne, \& Charles-Soverall, 2019), y la medición de los resultados e impactos incrementales relacionados con el desarrollo humano y la expectativa de las partes interesadas en la lucha contra la pobreza (Dwyer, 2007).

\section{Resultados}

Inicialmente, a partir de la revisión de la literatura, se identificaron ocho perfiles de grupos de interés a los cuales cualquier sector debe responder en relación con su impacto. A saber, colaboradores, clientes, cadena de abastecimiento, accionistas, medioambiente, gobierno y sociedad (Andriof \& McIntosh, 2017; Batista, 2018; Gupta \& Agrawal, 2016; Kim \& Kim, 2019; Lee, Seo, \& Sharma, 2013; Luo, Lam, Chau, Shen, \& Wang, 2017; Salmi \& Khan, 2019).

De este modo, conforme a lo presentado en el análisis competitivo realizado por la Unidad Administrativa Especial de Aeronáutica Civil, se decidió revisar los sitios web, así como los informes integrados, memorias de sostenibilidad o publicaciones relacionadas de Avianca, Aires LATAM y Viva Colombia, puesto que representan más del $90 \%$ del sector de transporte aéreo colombiano, dan de cuenta el comportamiento de este y permiten evaluar el accountability como herramienta de marketing relacional.

En ese sentido, se logró encontrar la información relacionada con el proceso de accountability entre el año 2012 y 2019 de las compañías Aires LATAM y Avianca; mientras que para Viva Colombia (Viva Air) solo existe una serie de hechos históricos en la página web que serán analizados a continuación. Por lo anteriormente mencionado, se revisaron 16 documentos y un espacio web, con el fin de caracterizar y evaluar el comportamiento del sector a partir de la matriz de observación y registro.

La matriz de observación y registro, como menciona el componente metodológico, se construyó basado en los principales hallazgos que aborda la literatura en la que se intervino principalmente las mejores prácticas de accountability planteadas dentro y fuera del sector; posteriormente se presentó a tres jueces expertos en el tema de la rendición de cuentas, quienes orientaron algunos aspectos relevantes partiendo de su experiencia en la aplicación de estándares GRI, reporte integrado, ISO 26000 y comunidad académica. Así bien, como resultado se definen los ocho factores relevantes, los cuales se representaron por medio de una serie de preguntas y afirmaciones. 
Accountability, marketing relacional y grupos de interés en el transporte aéreo colombiano...

\section{V.1 Factor 1 - información}

Una vez revisadas las memorias de sostenibilidad, la Tabla 1 resume el comportamiento sectorial relacionado con los aspectos evaluados dentro del factor de "Información". En este se identifica que el sector de transporte aéreo colombiano cumple con la divulgación de información financiera y dispone de un espacio en su página web para la comunicación de su estrategia de sostenibilidad. Aspectos como el aseguramiento de la información no financiera han sido adoptados conforme se han desarrollado nuevos estándares y requerimientos por los organismos internacionales, por lo que a partir del 2017 se estableció como mecanismo de transparencia para los líderes empresariales.

Es de resaltar que Viva Air presenta un comportamiento diferente al de las dos líderes, razón que conllevaría a concluir que, dentro de su estrategia relacional, la revelación de la información no se comparte de forma estructurada y por ende no separa los resultados de carácter financiero de los no financieros.

Así bien, del análisis cualitativo realizado se puede destacar que Avianca desde 2013 dispone sus memorias de sostenibilidad a procesos de verificación externa ante la Global Reporting Initiative. Sin embargo, el cambio de estándares llevó a que la compañía no verificara nuevamente con la firma Deloitte sino hasta 2016, año en el que decide compilar la información financiera y no financiera en un único documento.

Ahora bien, a partir de los elementos analizados en la matriz de observación y registro, se evidencia que la auditoria o aseguramiento de la información no financiera; así como, la contribución tributaria de la actividad desarrollada, son los principales elementos que sistemáticamente no se desarrollan en el sector.

Del mismo modo, LATAM desde 2012 ha buscado garantizar la transparencia de la información a través de procesos de verificación externa gracias a la firma Deloitte, por lo que divulga constantemente su plan estratégico a través de cifras de operación en 13 países.

Tabla 1. Observación del Factor 1 (Información)

\begin{tabular}{|c|c|c|c|c|c|c|c|c|c|}
\hline \multirow[b]{2}{*}{ 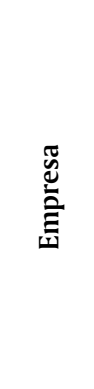 } & \multirow[b]{2}{*}{ Año } & \multicolumn{8}{|c|}{ Información } \\
\hline & & 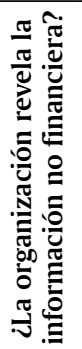 & 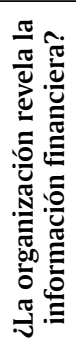 & 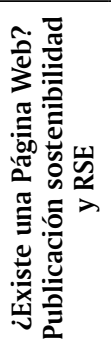 & 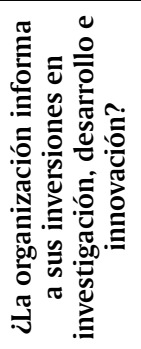 & 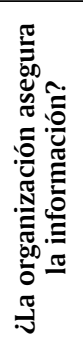 & 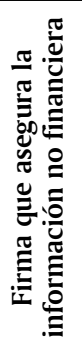 & 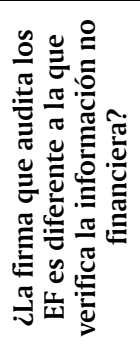 & 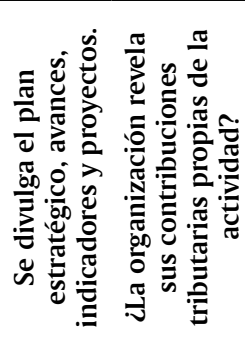 \\
\hline & 012 & $\checkmark 1$ & $\checkmark 1$ & $\checkmark 1$ & $\checkmark 1$ & $\times 0$ & $\times 0$ & $\checkmark 1$ & $\checkmark 1$ \\
\hline Latam & 2012 & $\checkmark 1$ & $\checkmark 1$ & $\checkmark 1$ & $\checkmark 1$ & $\checkmark 1$ & $\checkmark 1$ & $\checkmark 1$ & $\checkmark 1$ \\
\hline
\end{tabular}




\begin{tabular}{|c|c|c|c|c|c|c|c|c|c|c|}
\hline \multirow[b]{2}{*}{ 吢 } & \multirow[b]{2}{*}{ Año } & \multicolumn{9}{|c|}{ Información } \\
\hline & & 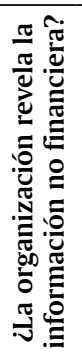 & 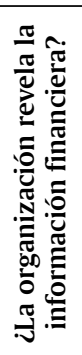 & 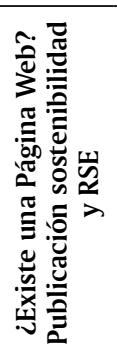 & 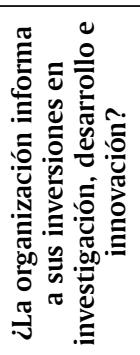 & 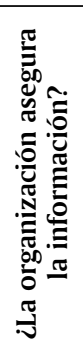 & 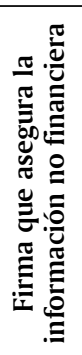 & 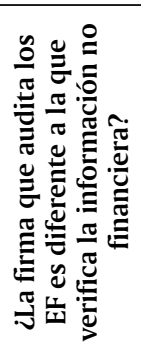 & 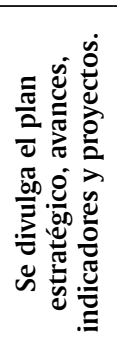 & 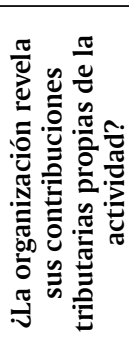 \\
\hline Avianca & 2013 & $\checkmark 1$ & $\checkmark 1$ & $\checkmark 1$ & $\checkmark 1$ & $\checkmark 1$ & $\checkmark 1$ & $\checkmark 1$ & $\checkmark 1$ & $\checkmark 1$ \\
\hline Latam & 2013 & $\checkmark 1$ & $\checkmark 1$ & $\checkmark 1$ & $\checkmark 1$ & $\checkmark 1$ & $\checkmark 1$ & $\checkmark 1$ & $\checkmark 1$ & $\checkmark 1$ \\
\hline Avianca & 2014 & $\checkmark 1$ & $\checkmark 1$ & $\checkmark 1$ & $\checkmark 1$ & $\times 0$ & $\times 0$ & $\checkmark 1$ & $\checkmark 1$ & $\checkmark 1$ \\
\hline Latam & 2014 & $\checkmark 1$ & $\checkmark 1$ & $\checkmark 1$ & $\checkmark 1$ & $\checkmark 1$ & $\checkmark 1$ & $\checkmark 1$ & $\checkmark 1$ & $\checkmark 1$ \\
\hline Avianca & 2015 & $\checkmark 1$ & $\checkmark 1$ & $\checkmark 1$ & $\checkmark 1$ & $\times 0$ & $\times 0$ & $\checkmark 1$ & $\checkmark 1$ & $\checkmark 1$ \\
\hline Latam & 2015 & $\checkmark 1$ & $\checkmark 1$ & $\checkmark 1$ & $\checkmark 1$ & $\checkmark 1$ & $\checkmark 1$ & $\checkmark 1$ & $\checkmark 1$ & $\times 0$ \\
\hline Avianca & 2016 & $\checkmark 1$ & $\checkmark 1$ & $\checkmark 1$ & $\checkmark 1$ & $\checkmark 1$ & $\times 0$ & $\checkmark 1$ & $\checkmark 1$ & $\checkmark 1$ \\
\hline Latam & 2016 & $\checkmark 1$ & $\checkmark 1$ & $\checkmark 1$ & $\checkmark 1$ & $\checkmark 1$ & $\checkmark 1$ & $\checkmark 1$ & $\checkmark 1$ & $\checkmark 1$ \\
\hline Avianca & 2017 & $\checkmark 1$ & $\checkmark 1$ & $\checkmark 1$ & $\checkmark 1$ & $\checkmark 1$ & $\checkmark 1$ & $\checkmark 1$ & $\checkmark 1$ & $\checkmark 1$ \\
\hline Latam & 2017 & $\checkmark 1$ & $\checkmark 1$ & $\checkmark 1$ & $\checkmark 1$ & $\checkmark 1$ & $\checkmark 1$ & $\checkmark 1$ & $\checkmark 1$ & $\times 0$ \\
\hline Avianca & 2018 & $\checkmark 1$ & $\checkmark 1$ & $\checkmark 1$ & $\checkmark 1$ & $\checkmark 1$ & $\checkmark 1$ & $\checkmark 1$ & $\checkmark 1$ & $\checkmark 1$ \\
\hline Latam & 2018 & $\checkmark 1$ & $\checkmark 1$ & $\checkmark \quad 1$ & $\checkmark 1$ & $\checkmark 1$ & $\checkmark \quad 1$ & $\checkmark 1$ & $\checkmark 1$ & $\checkmark 1$ \\
\hline Viva Air & s.f. & $\checkmark 1$ & $\times 0$ & $\checkmark \quad 1$ & $\times 0$ & $\times 0$ & $\times 0$ & $\times 0$ & $\times 0$ & $\times 0$ \\
\hline Avianca & 2019 & $\checkmark 1$ & $\checkmark 1$ & $\checkmark 1$ & $\checkmark 1$ & $\checkmark 1$ & $\checkmark 1$ & $\checkmark 1$ & $\checkmark 1$ & $\checkmark 1$ \\
\hline Latam & 2019 & $\checkmark 1$ & $\checkmark 1$ & $\checkmark \quad 1$ & $\times 0$ & $\checkmark 1$ & $\checkmark \quad 1$ & $\checkmark 1$ & $\checkmark 1$ & $\checkmark 1$ \\
\hline Viva Air & s.f. & $\checkmark 1$ & $\times 0$ & $\checkmark \quad 1$ & $\times 0$ & $\times 0$ & $\times 0$ & $\times 0$ & $\times 0$ & $\times 0$ \\
\hline
\end{tabular}

Fuente: Elaboración propia de los autores

\section{V.2 Factor 2 - accionistas}

La Tabla 2 resume el comportamiento sectorial relacionado con los aspectos evaluados dentro del factor de los "Accionistas", identificando así que el sector de transporte aéreo colombiano presenta una clara preocupación por rendir cuentas sobre sus estrategias y así proteger la lealtad con la marca, determinar los resultados económicos relevantes para los inversionistas (rendimientos, valor generado y redistribuido) y presentar los ejercicios de prospectiva sobre el sector y la competencia.

No obstante, se evidencia que la información presentada no es validada con el grupo de interés mediante diálogos confirmatorios de cumplimiento sobre este; así mismo, es evidente que las proyecciones económicas futuras sobre los resultados obtenidos han tomado relevancia en los últimos años, como mecanismo para mantener la confianza del inversionista sobre la organización. 
Accountability, marketing relacional y grupos de interés en el transporte aéreo colombiano...

Viva Air, por su parte, no presenta ningún tipo de información relacionada con las características evaluadas sobre su relación con los inversionistas. Así bien, del análisis cualitativo realizado se puede destacar que Avianca en sus informes presenta el cumplimiento de las metas propuestas a los accionistas en relación con sus productos, pues resaltan que “...el producto turístico Avianca Tours obtuvo un ingreso de 55 millones de USD, cifra que permitió cumplir en $104 \%$ con la meta presupuestada..." (2013, p. 39); así como, el reconocimiento de aspectos por mejorar en relación el crecimiento, ya que para 2013 “....representó una caída de 2,4 puntos en participación en el mercado doméstico debido a la ampliación del mismo y la presencia de nuevos competidores..." (2014, p. 33).

Del mismo modo, LATAM ha cambiado su estrategia de rendición de cuentas con los accionistas, gracias a la fusión de LAN y TAM en 2012, para posicionar una única marca comercial. Adicionalmente, plantea proyectos específicos anuales en relación con los resultados obtenidos, evidenciando así propuestas claras para aumentar el valor de los accionistas al “...invertir aproximadamente US\$750 millones en CAPEX no-flota durante 2019, incluyendo activos intangibles, mantenimiento, inversiones en motores y repuestos, así como la renovación de las cabinas de sus modelos Boeing 767, Boeing 777 y A320-Fam..." (2019, p. 63).

Tabla 2. Observación del Factor 2 (Accionistas)

\begin{tabular}{|c|c|c|c|c|c|c|c|c|}
\hline \multirow[b]{2}{*}{ 莺 } & \multirow[b]{2}{*}{ Año } & \multicolumn{7}{|c|}{ Accionistas } \\
\hline & & 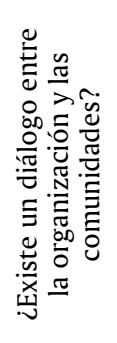 & 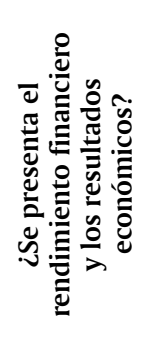 & 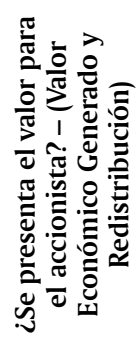 & 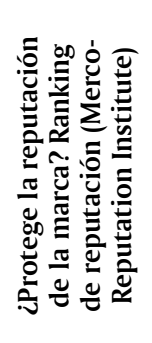 & 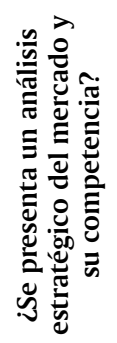 & 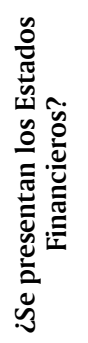 & 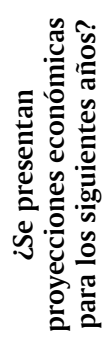 \\
\hline Avianca & 2012 & $\times 0$ & $\checkmark 1$ & $\checkmark 1$ & $\checkmark 1$ & $\checkmark 1$ & $\checkmark 1$ & $\times 0$ \\
\hline Latam & 2012 & $\times 0$ & $\checkmark 1$ & $\checkmark 1$ & $\checkmark 1$ & $\checkmark 1$ & $\checkmark 1$ & $\times 0$ \\
\hline Avianca & 2013 & $\times 0$ & $\checkmark 1$ & $\checkmark 1$ & $\checkmark 1$ & $\checkmark 1$ & $\checkmark 1$ & $\times 0$ \\
\hline Latam & 2013 & $\times 0$ & $\checkmark 1$ & $\checkmark 1$ & $\checkmark 1$ & $\checkmark 1$ & $\checkmark 1$ & $\times 0$ \\
\hline Avianca & 2014 & $\times 0$ & $\checkmark 1$ & $\checkmark 1$ & $\checkmark 1$ & $\times 0$ & $\checkmark 1$ & $\times 0$ \\
\hline Latam & 2014 & $\times 0$ & $\checkmark 1$ & $\checkmark 1$ & $\times 0$ & $\checkmark 1$ & $\checkmark 1$ & $\times 0$ \\
\hline Avianca & 2015 & $\times 0$ & $\checkmark 1$ & $\checkmark 1$ & $\checkmark 1$ & $\checkmark 1$ & $\checkmark 1$ & $\times 0$ \\
\hline Latam & 2015 & $\times 0$ & $\checkmark 1$ & $\checkmark 1$ & $\checkmark 1$ & $\checkmark 1$ & $\checkmark 1$ & $\times 0$ \\
\hline Avianca & 2016 & $\times 0$ & $\checkmark 1$ & $\checkmark 1$ & $\checkmark 1$ & $\checkmark 1$ & $\checkmark 1$ & $\checkmark 1$ \\
\hline Latam & 2016 & $\times 0$ & $\checkmark 1$ & $\checkmark 1$ & $\checkmark 1$ & $\checkmark 1$ & $\checkmark 1$ & $\times 0$ \\
\hline Avianca & 2017 & $\times 0$ & $\checkmark 1$ & $\checkmark 1$ & $\checkmark 1$ & $\checkmark 1$ & $\checkmark 1$ & $\checkmark 1$ \\
\hline Latam & 2017 & $\times 0$ & $\checkmark 1$ & $\checkmark 1$ & $\checkmark 1$ & $\checkmark 1$ & $\checkmark 1$ & $\checkmark 1$ \\
\hline
\end{tabular}




\begin{tabular}{|c|c|c|c|c|c|c|c|c|}
\hline \multirow[b]{2}{*}{$\begin{array}{l}\text { 苞 } \\
\text { 悹 }\end{array}$} & \multirow[b]{2}{*}{ Año } & \multicolumn{7}{|c|}{ Accionistas } \\
\hline & & 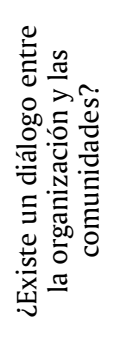 & 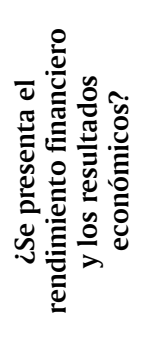 & 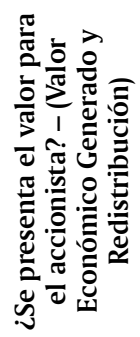 & 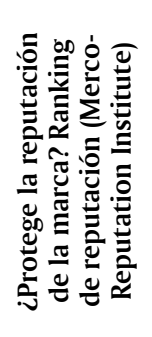 & 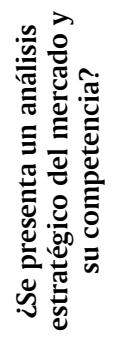 & 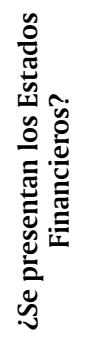 & 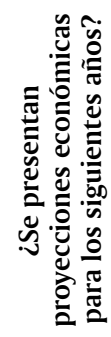 \\
\hline Avianca & 2018 & $\times 0$ & $\checkmark 1$ & $\checkmark 1$ & $\checkmark 1$ & $\checkmark 1$ & $\checkmark 1$ & $\begin{array}{lll} & 1\end{array}$ \\
\hline Latam & 2018 & $\times 0$ & $\checkmark 1$ & $\checkmark 1$ & $\checkmark 1$ & $\checkmark 1$ & $\checkmark 1$ & $\checkmark 1$ \\
\hline Viva Air & s.f. & $\times 0$ & $\times 0$ & $\times 0$ & $\times 0$ & $\times 0$ & $\times 0$ & $\times 0$ \\
\hline Avianca & 2019 & $\times 0$ & $\checkmark 1$ & $\checkmark 1$ & $\checkmark 1$ & $\times 0$ & $\checkmark 1$ & $\times 0$ \\
\hline Latam & 2019 & $\times 0$ & $\checkmark 1$ & $\checkmark 1$ & $\checkmark 1$ & $\checkmark 1$ & $\checkmark 1$ & $\times 0$ \\
\hline Viva Air & s.f. & $\times 0$ & $\times 0$ & $\times 0$ & $\times 0$ & $\times 0$ & $\times 0$ & $\times 0$ \\
\hline
\end{tabular}

Fuente: Elaboración propia de los autores

\section{V.3 Factor 3 - colaboradores}

La Tabla 3 resume el comportamiento sectorial relacionado con los aspectos evaluados dentro del factor de los "Colaboradores", identificando así que el sector en general revela información relevante sobre la contribución al cierre de brechas mediante programas de crecimiento profesional y escala al interior de la organización, programas y políticas de remuneración, bienestar del personal y retención laboral. Empero, cabe mencionar que no se evidencian iniciativas para confirmar si los resultados presentados son las expectativas de este grupo de interés.

Tabla 3. Observación del Factor 3 (Colaboradores)

\begin{tabular}{|c|c|c|c|c|c|c|c|c|c|}
\hline \multirow[b]{2}{*}{ 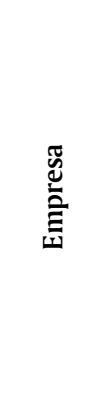 } & \multirow[b]{2}{*}{ Año } & \multicolumn{8}{|c|}{ Empleados } \\
\hline & & 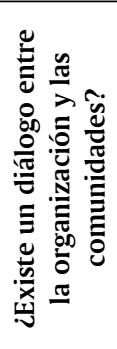 & 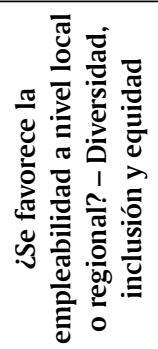 & 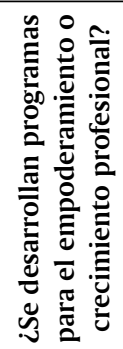 & 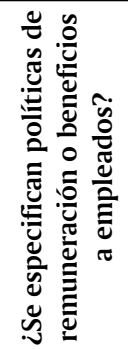 & 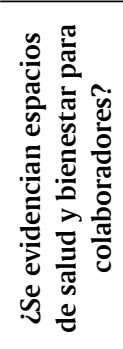 & 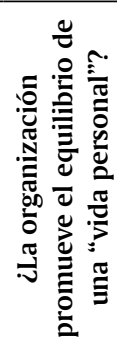 & 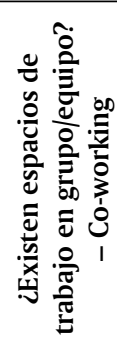 & 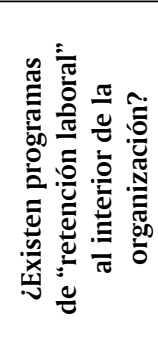 \\
\hline Avianca & 2012 & $\times 0$ & $\checkmark 1$ & $\checkmark 1$ & $\checkmark 1$ & $\checkmark 1$ & $\checkmark 1$ & $\checkmark 1$ & $\checkmark 1$ \\
\hline Latam & 2012 & $\times 0$ & $\checkmark 1$ & $\checkmark 1$ & $\checkmark 1$ & $\checkmark 1$ & $\checkmark 1$ & $\checkmark 1$ & $\checkmark 1$ \\
\hline Avianca & 2013 & $\times 0$ & $\checkmark 1$ & $\checkmark 1$ & $\times 0$ & $\checkmark 1$ & $\checkmark 1$ & $\times 0$ & $\checkmark 1$ \\
\hline Latam & 2013 & $\times 0$ & $\checkmark 1$ & $\checkmark 1$ & $\checkmark 1$ & $\checkmark 1$ & $\times 0$ & $\times 0$ & $\checkmark 1$ \\
\hline Avianca & 2014 & $\times 0$ & $\checkmark 1$ & $\checkmark 1$ & $\checkmark 1$ & $\checkmark 1$ & $\checkmark 1$ & $\checkmark 1$ & $\checkmark 1$ \\
\hline
\end{tabular}




\begin{tabular}{|c|c|c|c|c|c|c|c|c|c|}
\hline \multirow[b]{2}{*}{ 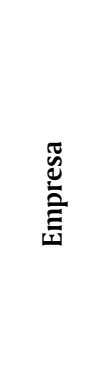 } & \multirow[b]{2}{*}{ Año } & \multicolumn{8}{|c|}{ Empleados } \\
\hline & & 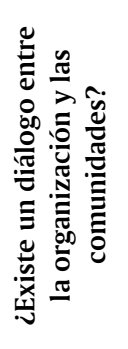 & 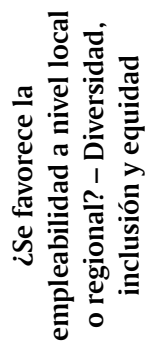 & 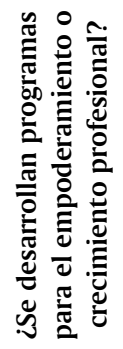 & 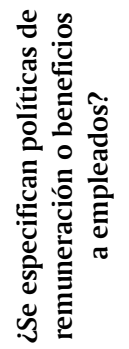 & 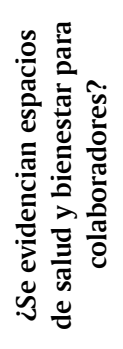 & 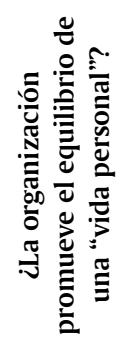 & 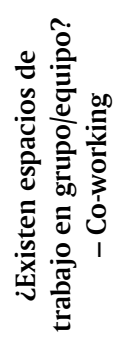 & 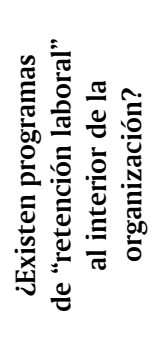 \\
\hline Latam & 2014 & $\times 0$ & $\checkmark 1$ & $\checkmark 1$ & $\checkmark 1$ & $\checkmark 1$ & $\checkmark 1$ & $\times 0$ & $\checkmark 1$ \\
\hline Avianca & 2015 & $\times 0$ & $\checkmark 1$ & $\checkmark 1$ & $\times 0$ & $\checkmark 1$ & $\checkmark 1$ & $\checkmark 1$ & $\checkmark 1$ \\
\hline Latam & 2015 & $\times 0$ & $\checkmark 1$ & $\checkmark 1$ & $\checkmark 1$ & $\checkmark 1$ & $\checkmark 1$ & $\checkmark 1$ & $\checkmark 1$ \\
\hline Avianca & 2016 & $\times 0$ & $\checkmark 1$ & $\checkmark 1$ & $\checkmark 1$ & $\checkmark 1$ & $\checkmark 1$ & $\checkmark 1$ & $\checkmark 1$ \\
\hline Latam & 2016 & $\times 0$ & $\checkmark 1$ & $\checkmark 1$ & $\checkmark 1$ & $\checkmark 1$ & $\times 0$ & $\checkmark 1$ & $\checkmark 1$ \\
\hline Avianca & 2017 & $\times 0$ & $\checkmark 1$ & $\checkmark 1$ & $\checkmark 1$ & $\checkmark 1$ & $\checkmark 1$ & $\checkmark 1$ & $\checkmark 1$ \\
\hline Latam & 2017 & $\times 0$ & $\checkmark 1$ & $\checkmark 1$ & $\checkmark 1$ & $\checkmark 1$ & $\checkmark 1$ & $\checkmark 1$ & $\checkmark 1$ \\
\hline Avianca & 2018 & $\times 0$ & $\checkmark 1$ & $\checkmark 1$ & $\checkmark 1$ & $\checkmark 1$ & $\checkmark 1$ & $\checkmark 1$ & $\checkmark 1$ \\
\hline Latam & 2018 & $\times 0$ & $\checkmark 1$ & $\checkmark 1$ & $\checkmark 1$ & $\checkmark 1$ & $\checkmark 1$ & $\checkmark 1$ & $\checkmark 1$ \\
\hline Viva Air & s.f. & $\times 0$ & $\times 0$ & $\checkmark 1$ & $\times 0$ & $\times 0$ & $\times 0$ & $\checkmark 1$ & $\times 0$ \\
\hline Avianca & 2019 & $\times 0$ & $\checkmark 1$ & $\checkmark 1$ & $\checkmark 1$ & $\checkmark 1$ & $\checkmark 1$ & $\checkmark 1$ & $\checkmark 1$ \\
\hline Latam & 2019 & $\times 0$ & $\times 0$ & $\times 0$ & $\checkmark 1$ & $\times 0$ & $\checkmark 1$ & $\times 0$ & $\checkmark 1$ \\
\hline Viva Air & s.f. & $\times 0$ & $\times 0$ & $\times 0$ & $\times 0$ & $\times 0$ & $\times 0$ & $\times 0$ & $\times 0$ \\
\hline
\end{tabular}

Fuente: Elaboración propia de los autores

Por su parte, Viva Air enmarca que “...a través de la democratización de la aviación se promueve el empoderamiento de las comunidades en diferentes ambientes con el fin de fomentar el trabajo en equipo, la solidaridad y el respeto por el otro..." (s.f.), lo que permitiría identificar esfuerzos por incentivar el trabajo en equipo al interior de la organización. Así bien, del análisis cualitativo realizado también se puede destacar que Avianca se ha posicionado como líder en equidad salarial ya que "...tienen un esquema salarial establecido por cargos, que no tiene en cuenta el género de la persona que desempeña cada labor...” (2013, p. 77). Esta organización busca el equilibrio de la vida de sus trabajadores mediante el “... equilibrio entre la vida laboral y la vida personal, se fomentó la participación de los colaboradores en los torneos deportivos, ferias, recargas motivacionales, programas de recreación y la semana artística, entre otros...” (2013, p. 94).

Del mismo modo, LATAM muestra que desde 2012 ha implementado una cultura de "...contar con colaboradores de diversas procedencias, al tener presencia en distintos países de la región. Así, hoy en día sus colaboradores provienen de 50 nacionalidades diferentes..." (2013, p. 72), los cuales al interior de la organización tienen la oportunidad de formarse, crecer profesionalmente y ascender en las líneas de mando ya que en el transcurrir, esta aerolínea 
sigue “...expandiendo los movimientos internos, horizontales y verticales, gracias a los resultados del proceso de evaluación de los empleados. De todas las vacantes en cargos de mando medio y cargos ejecutivos que hubo en 2018, el $72 \%$ han sido ocupadas internamente...” (2019, p. 87).

\section{V.4 Factor 4 - consumidores}

La Tabla 4 resume el comportamiento sectorial relacionado con los aspectos evaluados dentro del factor de los "Consumidores", identificando así que el sector se disipa frente al nivel de rendición cuentas históricamente presentado. Lo anterior se evidencia puesto que antes de 2016, la mayoría de los ítems relacionados con el servicio y su calidad se encontraban ausentes en este tipo de informes, mientras que desde ese mismo año en adelante hay un mayor cumplimiento en la información revelada.

No obstante, una de las estrategias relacionales que más se ha posicionado dentro del sector, es la búsqueda por dar acceso al servicio mediante una propuesta para todos los niveles socioeconómicos; así como, la digitalización y conectividad en la prestación del servicio (pre-core, core y post-core). Cabe anotar que, dentro de los elementos por mejorar, según los asuntos materiales de este grupo de interés, se hace necesario que las organizaciones de este sector tomen la iniciativa de informar sobre los indicadores de calidad e higiene de la prestación del servicio.

Tabla 4. Observación del Factor 4 (Consumidores)

\begin{tabular}{|c|c|c|c|c|c|c|c|c|c|}
\hline \multirow[b]{2}{*}{ 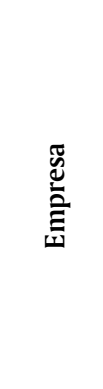 } & \multirow[b]{2}{*}{ Año } & \multicolumn{8}{|c|}{ Consumidor } \\
\hline & & 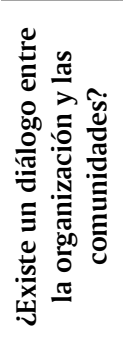 & 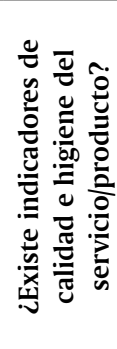 & 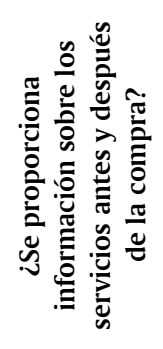 & 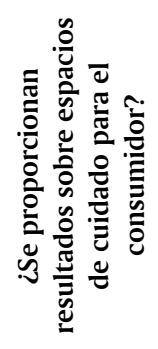 & 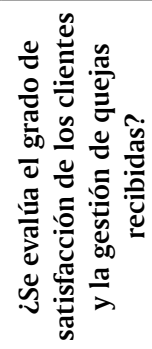 & 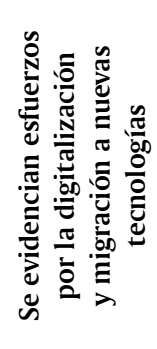 & 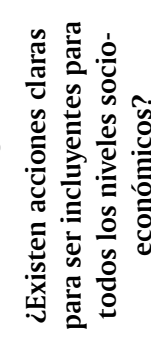 & 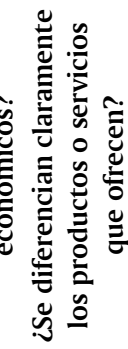 \\
\hline Avianca & 2012 & $\times 0$ & $\times 0$ & $\times 0$ & $\checkmark 1$ & $\times 0$ & $\checkmark 1$ & $\checkmark 1$ & $\checkmark 1$ \\
\hline Latam & 2012 & $\times 0$ & $\checkmark 1$ & $\checkmark 1$ & $\checkmark 1$ & $\checkmark 1$ & $\times 0$ & $\times 0$ & $\checkmark 1$ \\
\hline Avianca & 2013 & $\times 0$ & $\times 0$ & $\times 0$ & $\checkmark 1$ & $\times 0$ & $\times 0$ & $\checkmark 1$ & $\checkmark 1$ \\
\hline Latam & 2013 & $\times 0$ & $\checkmark 1$ & $\times 0$ & $\checkmark 1$ & $\checkmark 1$ & $\checkmark 1$ & $\times 0$ & $\checkmark 1$ \\
\hline Avianca & 2014 & $\times 0$ & $\times 0$ & $\times 0$ & $\checkmark 1$ & $\checkmark 1$ & $\checkmark 1$ & $\checkmark 1$ & $\checkmark 1$ \\
\hline Latam & 2014 & $\times 0$ & $\checkmark 1$ & $\times 0$ & $\times 0$ & $\checkmark 1$ & $\checkmark 1$ & $\checkmark 1$ & $\checkmark 1$ \\
\hline Avianca & 2015 & $\times 0$ & $\times 0$ & $\times 0$ & $\checkmark 1$ & $\times 0$ & $\times 0$ & $\checkmark 1$ & $\times 0$ \\
\hline Latam & 2015 & $\times 0$ & $\times 0$ & $\times 0$ & $\times 0$ & $\checkmark 1$ & $\checkmark 1$ & $\checkmark 1$ & $\checkmark 1$ \\
\hline Avianca & 2016 & $\times 0$ & $\checkmark 1$ & $\checkmark 1$ & $\checkmark 1$ & $\checkmark 1$ & $\checkmark 1$ & $\checkmark 1$ & $\checkmark 1$ \\
\hline
\end{tabular}




\begin{tabular}{|c|c|c|c|c|c|c|c|c|c|}
\hline \multirow[b]{2}{*}{ 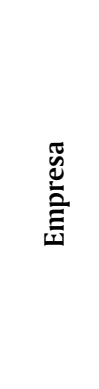 } & \multirow[b]{2}{*}{ Año } & \multicolumn{8}{|c|}{ Consumidor } \\
\hline & & 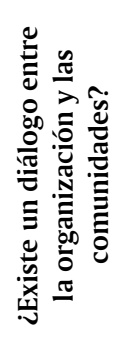 & 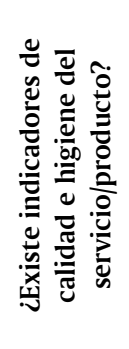 & 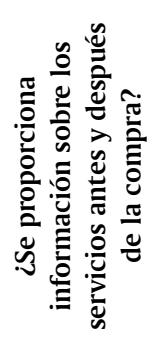 & 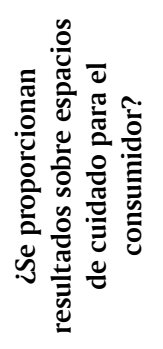 & 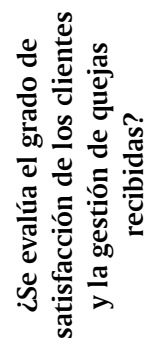 & 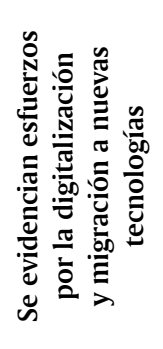 & 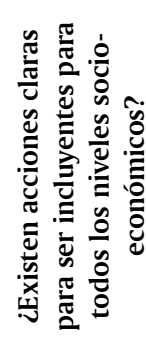 & 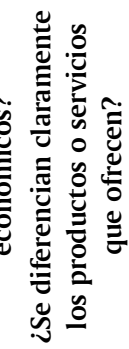 \\
\hline Latam & 2016 & $\times 0$ & $\times 0$ & $\times 0$ & $\times 0$ & $\checkmark 1$ & $\checkmark 1$ & $\checkmark 1$ & $\checkmark 1$ \\
\hline Avianca & 2017 & $\times 0$ & $\checkmark 1$ & $\checkmark 1$ & $\checkmark 1$ & $\checkmark 1$ & $\checkmark 1$ & $\checkmark 1$ & $\checkmark 1$ \\
\hline Latam & 2017 & $\times 0$ & $\checkmark 1$ & $\times 0$ & $\checkmark 1$ & $\checkmark 1$ & $\checkmark 1$ & $\checkmark 1$ & $\checkmark 1$ \\
\hline Avianca & 2018 & $\times 0$ & $\checkmark 1$ & $\checkmark 1$ & $\checkmark 1$ & $\checkmark 1$ & $\checkmark 1$ & $\checkmark 1$ & $\checkmark 1$ \\
\hline Latam & 2018 & $\times 0$ & $\checkmark 1$ & $\checkmark 1$ & $\times 0$ & $\checkmark 1$ & $\checkmark 1$ & $\checkmark 1$ & $\checkmark 1$ \\
\hline Viva Air & s.f. & $\times 0$ & $\times 0$ & $\times 0$ & $\times 0$ & $\times 0$ & $\times 0$ & $\checkmark 1$ & $\times 0$ \\
\hline Avianca & 2019 & $\times 0$ & $\checkmark 1$ & $\checkmark 1$ & $\checkmark 1$ & $\checkmark 1$ & $\checkmark 1$ & $\checkmark 1$ & $\checkmark 1$ \\
\hline Latam & 2019 & $\times 0$ & $\checkmark 1$ & $\checkmark 1$ & $\checkmark 1$ & $\checkmark 1$ & $\checkmark 1$ & $\checkmark 1$ & $\times 0$ \\
\hline Viva Air & s.f. & $\times 0$ & $\times 0$ & $\times 0$ & $\times 0$ & $\times 0$ & $\times 0$ & $\times 0$ & $\times 0$ \\
\hline
\end{tabular}

Fuente: Elaboración propia de los autores

Así mismo, es de resaltar que Viva Air ha establecido como objetivo el “... querer democratizar la aviación porque en Viva Air están convencidos que viajar debe ser para todos..." (s.f.). Así bien, del análisis cualitativo realizado también se puede destacar que Avianca tuvo que buscar la unificación de su marca comercial en 2013 para que hacía 2016 pudiera integrar la tecnología y el enfoque en el cliente, evidenciado principalmente en la “...transformación a una organización líder en el campo digital, a través de la innovación, por lo que en 2016 define las funcionalidades e ideas digitales que le permitirán convertirse en una aerolínea digital, líder en la industria, que vuela aviones...” (2017, p. 88).

Del mismo modo, LATAM desde el 2012 ha buscado fortalecer un área especial de Servicio al Cliente para "...gestionar aquellas sugerencias, solicitudes, quejas y reclamos que recibamos y que no encuentren una solución en la experiencia regular de acuerdo con lo comprometido en la promesa de venta..." (2013, p. 45); de modo que para el 2018 "...el Grupo continúa avanzando con otras medidas para empoderar al pasajero y mejorar su experiencia con la marca, como por ejemplo, a través de desarrollo digital, inversiones en aeropuerto y entretenimiento a bordo..." (2019, p.12).

\section{V.5 Factor 5 - medioambiente}

La Tabla 5 resume el comportamiento sectorial relacionado con los aspectos evaluados dentro del factor de "Medioambiente", identificando así que el sector se encuentra altamente comprometido con este tema fundamental de sosteni- 
bilidad. Lo anterior, debido al seguimiento periódico que realizan las aerolíneas sobre su huella de carbono, gestión de residuos, conservación del agua y optimización energética. No obstante, aún se evidencian retos en relación con la verificación realizada por externos sobre los resultados y cumplimiento en los informes técnicos de sostenibilidad, un seguimiento real al impacto sobre el ruido generado, un esfuerzo por preservar la biodiversidad y generar capacidades para corroborar las expectativas con los stakeholders.

Viva Air, por su parte, no muestra una estrategia clara de relacionamiento de este tema fundamental con los grupos de interés. Por otro lado, del análisis cualitativo realizado se destaca que Avianca al interior del país ha optado por renovar su flota aérea de modo que “...permite a las compañías operativas de esta línea de negocio, ser eficientes en el consumo de combustible, reducir las emisiones de $\mathrm{CO} 2$, el ruido y generar menos residuos peligrosos en sus procesos de mantenimiento..." (2013, p. 52); cabe anotar que también divulga sus impactos negativos y estrategias para mitigar sus consecuencias, de modo que "...en los procesos de lavado de piezas y componentes...las compañías subsidiarias generaron agua contaminada, la cual recibe tratamiento físico-químico, microbiológico y disposición final adecuada por parte de organizaciones externas que cuentan con los permisos requeridos..." (2015, p. 90).

Tabla 5. Observación del Factor 5 (Medioambiente)

\begin{tabular}{|c|c|c|c|c|c|c|c|c|c|c|}
\hline \multirow[b]{2}{*}{ 总 } & \multirow[b]{2}{*}{ Año } & \multicolumn{9}{|c|}{ Medioambiente } \\
\hline & & 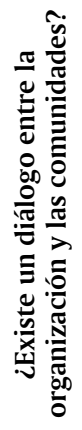 & 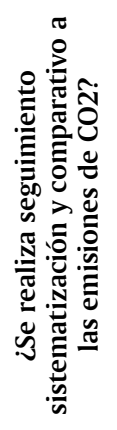 & 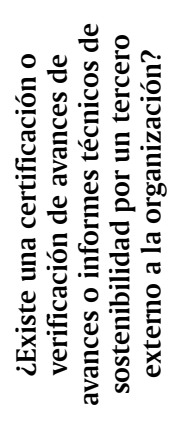 & 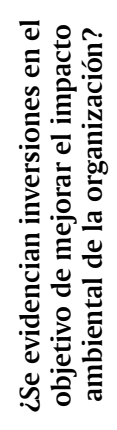 & 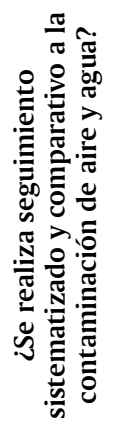 & 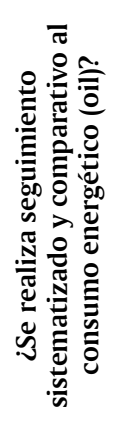 & 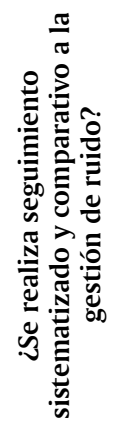 & 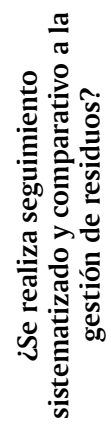 & 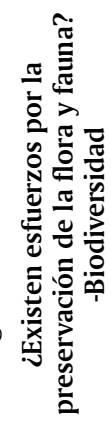 \\
\hline Avianca & 2012 & $\times 0$ & $\checkmark 1$ & $\times 0$ & $\checkmark 1$ & $\checkmark 1$ & $\checkmark 1$ & $\times 0$ & $\checkmark 1$ & $\times 0$ \\
\hline Latam & 2012 & $\times 0$ & $\checkmark 1$ & $\times 0$ & $\checkmark 1$ & $\checkmark 1$ & $\checkmark 1$ & $\checkmark 1$ & $\checkmark 1$ & $\checkmark 1$ \\
\hline Avianca & 2013 & $\times 0$ & $\checkmark 1$ & $\times 0$ & $\checkmark 1$ & $\checkmark 1$ & $\checkmark 1$ & $\times 0$ & $\checkmark 1$ & $\times 0$ \\
\hline Latam & 2013 & $\times 0$ & $\checkmark 1$ & $\checkmark 1$ & $\checkmark 1$ & $\checkmark 1$ & $\checkmark 1$ & $\times 0$ & $\checkmark 1$ & $\checkmark 1$ \\
\hline Avianca & 2014 & $\times 0$ & $\checkmark 1$ & $\times 0$ & $\checkmark 1$ & $\checkmark 1$ & $\checkmark 1$ & $\times 0$ & $\checkmark 1$ & $\times 0$ \\
\hline Latam & 2014 & $\times 0$ & $\checkmark 1$ & $\checkmark 1$ & $\checkmark 1$ & $\checkmark 1$ & $\checkmark 1$ & $\times 0$ & $\checkmark 1$ & $\checkmark 1$ \\
\hline Avianca & 2015 & $\times 0$ & $\checkmark 1$ & $\times 0$ & $\checkmark 1$ & $\checkmark 1$ & $\checkmark 1$ & $\times 0$ & $\checkmark 1$ & $\times 0$ \\
\hline Latam & 2015 & $\times 0$ & $\checkmark 1$ & $\checkmark 1$ & $\checkmark 1$ & $\checkmark 1$ & $\checkmark 1$ & $\checkmark 1$ & $\checkmark 1$ & $\times 0$ \\
\hline Avianca & 2016 & $\times 0$ & $\checkmark 1$ & $\times 0$ & $\checkmark 1$ & $\checkmark 1$ & $\checkmark 1$ & $\times 0$ & $\checkmark 1$ & $\times 0$ \\
\hline
\end{tabular}




\begin{tabular}{|c|c|c|c|c|c|c|c|c|c|c|}
\hline \multirow[b]{2}{*}{ 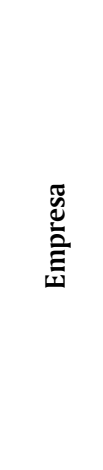 } & \multirow[b]{2}{*}{ Año } & \multicolumn{9}{|c|}{ Medioambiente } \\
\hline & & 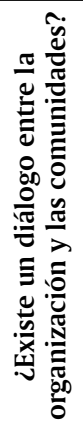 & 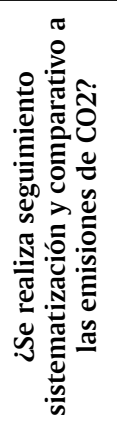 & 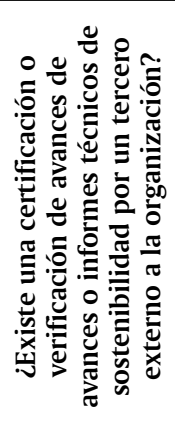 & 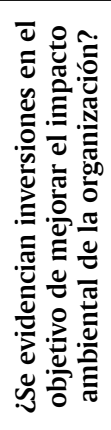 & 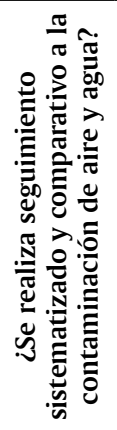 & 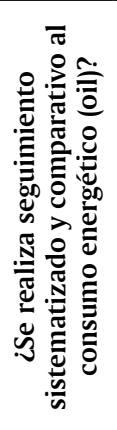 & 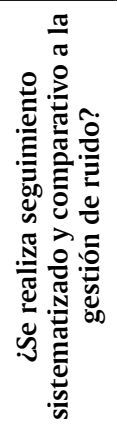 & 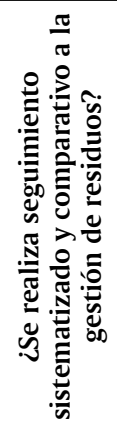 & 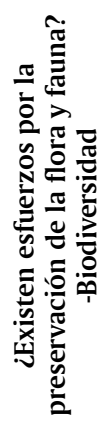 \\
\hline Latam & 2016 & $\times 0$ & $\checkmark 1$ & $\checkmark 1$ & $\checkmark 1$ & $\checkmark 1$ & $\checkmark 1$ & $\checkmark 1$ & $\checkmark 1$ & $\checkmark 1$ \\
\hline Avianca & 2017 & $\times 0$ & $\checkmark 1$ & $\times 0$ & $\checkmark 1$ & $\checkmark 1$ & $\checkmark 1$ & $\times 0$ & $\checkmark 1$ & $\times 0$ \\
\hline Latam & 2017 & $\times 0$ & $\checkmark 1$ & $\times 0$ & $\checkmark 1$ & $\checkmark 1$ & $\checkmark 1$ & $\times 0$ & $\checkmark 1$ & $\checkmark 1$ \\
\hline Avianca & 2018 & $\times 0$ & $\checkmark 1$ & $\times 0$ & $\checkmark 1$ & $\checkmark 1$ & $\checkmark 1$ & $\times 0$ & $\checkmark 1$ & $\checkmark 1$ \\
\hline Latam & 2018 & $\times 0$ & $\checkmark 1$ & $\checkmark 1$ & $\checkmark 1$ & $\checkmark 1$ & $\checkmark 1$ & $\times 0$ & $\checkmark 1$ & $\checkmark 1$ \\
\hline Viva Air & s.f. & $\times 0$ & $\times 0$ & $\times 0$ & $\times 0$ & $\times 0$ & $\times 0$ & $\times 0$ & $\times 0$ & $\times 0$ \\
\hline Avianca & 2019 & $\times 0$ & $\checkmark 1$ & $\checkmark 1$ & $\checkmark 1$ & $\checkmark 1$ & $\checkmark 1$ & $\times 0$ & $\checkmark 1$ & $\checkmark 1$ \\
\hline Latam & 2019 & $\times 0$ & $\checkmark 1$ & $\times 0$ & $\checkmark 1$ & $\checkmark 1$ & $\checkmark 1$ & $\checkmark 1$ & $\checkmark 1$ & $\checkmark 1$ \\
\hline Viva Air & s.f. & $\times 0$ & $\times 0$ & $\times 0$ & $\times 0$ & $\times 0$ & $\times 0$ & $\times 0$ & $\times 0$ & $\times 0$ \\
\hline
\end{tabular}

Fuente: Elaboración propia de los autores

Del mismo modo, LATAM desde 2012 ha buscado fortalecer su operación por medio de inversiones especializadas para mitigar sus impactos medioambientales, puesto que han actualizado su flota de Boeing 787 aprovechando que “... las ventajas medioambientales de estos aviones son la generación de hasta $20 \%$ menos emisiones de $\mathrm{CO} 2$ que aviones similares y la reducción de hasta un $40 \%$ en la huella de ruido..." (2013, p. 60); resultados que les ha permitido obtener en 2018 la "...certificación IEnvA (IATA Environmental Assessment) de las operaciones áreas, oficinas corporativas y MRO (Base de Mantenimiento) de LATAM Colombia, lo que hizo de la filial la única aerolínea del país en contar con esta certificación...” (2019, p. 76).

\section{V.6 Factor 6 - proveedores}

La Tabla 6 resume el comportamiento sectorial relacionado con los aspectos evaluados dentro del factor de los "Proveedores", identificando así que el sector da un lugar especial a su cadena de suministro y se enfoca en el cumplimiento de requisitos mínimos de sostenibilidad. Esto se evidencia a partir de las iniciativas por establecer auditorias de cumplimiento, estándares de evaluación de riesgos y manuales de abastecimiento, dentro de los que se encuentran la adopción de factores ambientales, sociales, de derechos humanos y la preocupación por el desarrollo local de sus comunidades. 
Tabla 6. Observación del Factor 6 (Proveedores)

\begin{tabular}{|c|c|c|c|c|c|c|c|}
\hline \multirow[b]{2}{*}{ 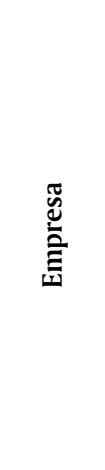 } & \multirow[b]{2}{*}{ Año } & \multicolumn{6}{|c|}{ Proveedores } \\
\hline & & 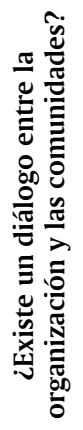 & 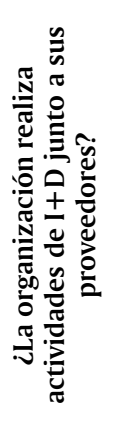 & 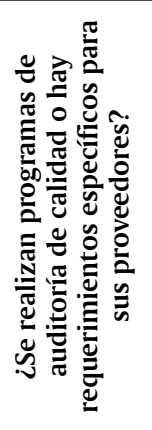 & 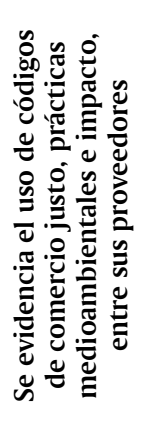 & 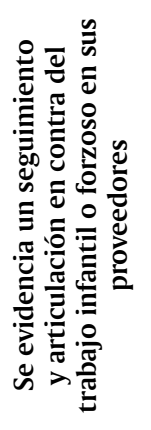 & 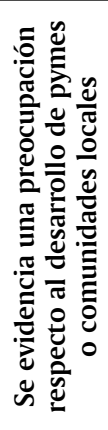 \\
\hline Avianca & 2012 & $\times 0$ & $\times 0$ & $\checkmark 1$ & $\checkmark 1$ & $\checkmark 1$ & $\checkmark 1$ \\
\hline Latam & 2012 & $\times 0$ & $\times 0$ & $\times 0$ & $\times 0$ & $\times 0$ & $\checkmark 1$ \\
\hline Avianca & 2013 & $\times 0$ & $\times 0$ & $\checkmark 1$ & $\checkmark 1$ & $\checkmark 1$ & $\checkmark 1$ \\
\hline Latam & 2013 & $\times 0$ & $\times 0$ & $\checkmark 1$ & $\checkmark 1$ & $\checkmark 1$ & $\checkmark 1$ \\
\hline Avianca & 2014 & $\times 0$ & $\times 0$ & $\checkmark 1$ & $\checkmark 1$ & $\checkmark 1$ & $\checkmark 1$ \\
\hline Latam & 2014 & $\times 0$ & $\times 0$ & $\checkmark 1$ & $\checkmark 1$ & $\checkmark 1$ & $\checkmark 1$ \\
\hline Avianca & 2015 & $\times 0$ & $\checkmark 1$ & $\checkmark 1$ & $\checkmark 1$ & $\checkmark 1$ & $\checkmark 1$ \\
\hline Latam & 2015 & $\times 0$ & $\times 0$ & $\checkmark 1$ & $\checkmark 1$ & $\checkmark 1$ & $\times 0$ \\
\hline Avianca & 2016 & $\times 0$ & $\times 0$ & $\checkmark 1$ & $\checkmark 1$ & $\checkmark 1$ & $\checkmark 1$ \\
\hline Latam & 2016 & $\times 0$ & $\times 0$ & $\checkmark 1$ & $\checkmark 1$ & $\checkmark 1$ & $\checkmark 1$ \\
\hline Avianca & 2017 & $\times 0$ & $\times 0$ & $\checkmark 1$ & $\checkmark 1$ & $\checkmark 1$ & $\times 0$ \\
\hline Latam & 2017 & $\times 0$ & $\times 0$ & $\checkmark 1$ & $\checkmark 1$ & $\checkmark 1$ & $\checkmark 1$ \\
\hline Avianca & 2018 & $\times 0$ & $\times 0$ & $\checkmark 1$ & $\checkmark 1$ & $\checkmark 1$ & $\times 0$ \\
\hline Latam & 2018 & $\times 0$ & $\times 0$ & $\checkmark 1$ & $\checkmark 1$ & $\checkmark 1$ & $\times 0$ \\
\hline Viva Air & s.f. & $\times 0$ & $\times 0$ & $\times 0$ & $\times 0$ & $\times 0$ & $\times 0$ \\
\hline Avianca & 2019 & $\times 0$ & $\times 0$ & $\checkmark 1$ & $\checkmark 1$ & $\times 0$ & $\times 0$ \\
\hline Latam & 2019 & $\times 0$ & $\times 0$ & $\times 0$ & $\checkmark 1$ & $\times 0$ & $\checkmark 1$ \\
\hline Viva Air & s.f. & $\times 0$ & $\times 0$ & $\times 0$ & $\times 0$ & $\checkmark 1$ & $\times 0$ \\
\hline
\end{tabular}

Fuente: Elaboración propia de los autores

Es de resaltar que en esta industria los procesos de investigación, desarrollo e innovación son concentrados en los fabricantes aeronáuticos y por ende es uno de los criterios menos relevantes en la rendición de cuentas, por su naturaleza oligopólica. No obstante; con la globalización de las compañías que pertenecen a este sector, se evidencia claramente una perdida por el enfoque de proveeduría local en los últimos años.

Por otro lado, Viva Air no presenta información relacionada con su interacción con Proveedores. No obstante, del análisis cualitativo realizado se destaca que Avianca realiza verificaciones de cumplimiento por parte de sus proveedores y “...para las empresas que tuvieron calificación menor a $65 \%$, se establecen 
planes de mejora y se hace seguimiento a los mismos..." (2014, p. 52), iniciativa que para 2018 se ha reformulado en el "...Manual de Proveedores, con el fin de consolidar los lineamientos de la Compañía e informar a nuestros proveedores los mecanismos de control, monitoreo y gestión de nuestra cadena de abastecimiento sostenible..." (2019, p. 168).

Del mismo modo, LATAM en 2012 se compromete con el bienestar de las comunidades locales mediante el objetivo de promover "...el desarrollo económico de la zona donde operan. Por esta razón, privilegian el aprovisionamiento desde empresas que operan en la región...” (2013, p. 50); sin embargo, con el fortalecimiento ético de la compañía, las políticas de abastecimiento se basan en el "...seguimiento a sospechas en casos de competencia desleal, esquemas de corrupción, financiamiento al terrorismo y narcotráfico, explotación de trabajo infantil o análogo al del esclavo e inconformidades ambientales, LATAM se vale de un sistema de consulta de bases..." (2019, p. 667).

\section{V.7 Factor 7 - gobernanza}

La Tabla 7 resume el comportamiento sectorial relacionado con los aspectos evaluados dentro del factor de "Gobernanza", identificando así que el sector de transporte aéreo colombiano ha dado una amplia revisión a la implementación de estructuras de gobierno corporativo, independencia entre los órganos de decisión y el establecimiento de comités consultivos especializados en temas relacionados con el desarrollo empresarial. Así mismo, los agentes del sector se han enfocado en el desarrollo de Códigos de Ética y a partir de 2015 han incluido aspectos relacionados con el control de fraude y lavado de activos.

Es de resaltar que en muy pocos informes se hace referencia a la constitución de un órgano consultivo especializado en los temas medioambientales, de modo que se puede observar que las estrategias de mitigación de impacto están en cabeza del presidente de las compañías.

Tabla 7. Observación del Factor 7 (Gobernanza)

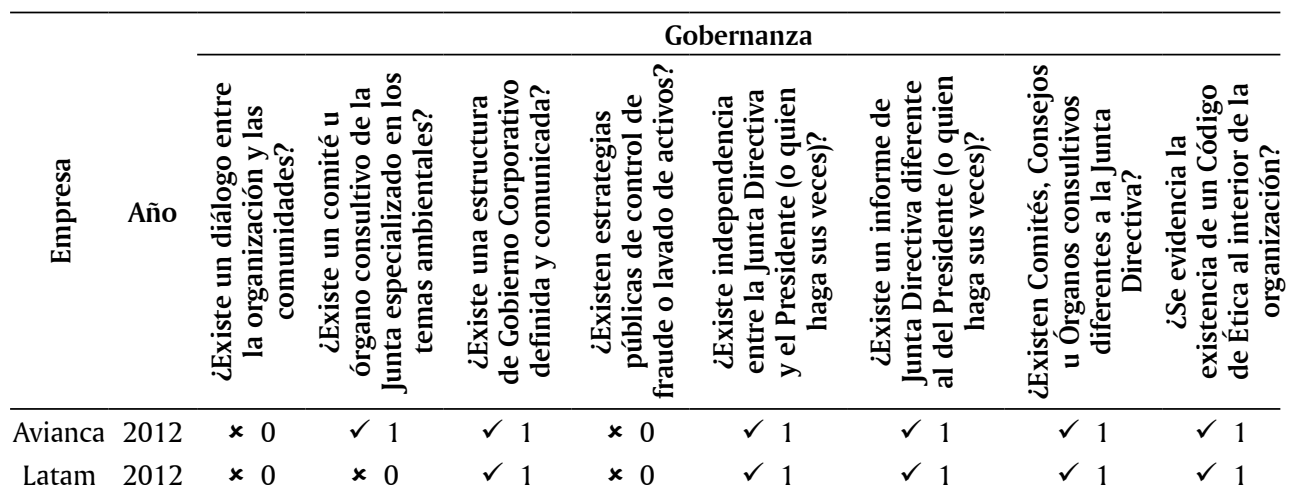




\begin{tabular}{|c|c|c|c|c|c|c|c|c|c|}
\hline \multirow[b]{2}{*}{ 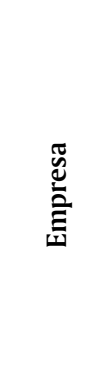 } & \multirow[b]{2}{*}{ Año } & \multicolumn{8}{|c|}{ Gobernanza } \\
\hline & & 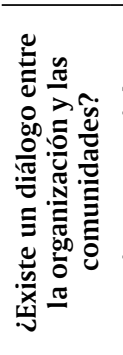 & 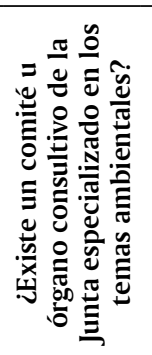 & 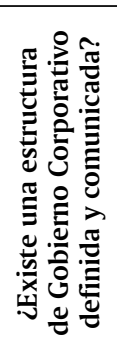 & 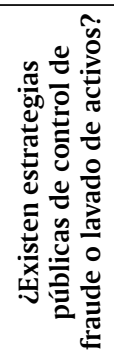 & 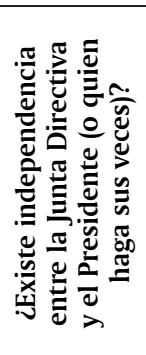 & 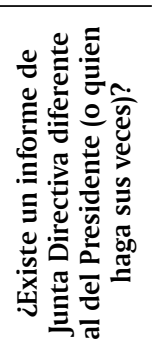 & 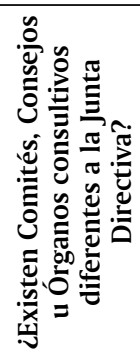 & 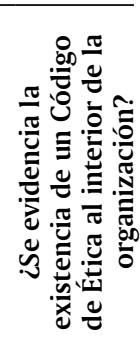 \\
\hline Avianca & 2013 & $\times 0$ & $\checkmark 1$ & $\checkmark 1$ & $\times 0$ & $\checkmark 1$ & $\checkmark 1$ & $\checkmark 1$ & $\checkmark 1$ \\
\hline Latam & 2013 & $\times 0$ & $\checkmark 1$ & $\checkmark 1$ & $\times 0$ & $\checkmark 1$ & $\checkmark 1$ & $\checkmark 1$ & $\checkmark 1$ \\
\hline Avianca & 2014 & $\times 0$ & $\times 0$ & $\checkmark 1$ & $\times 0$ & $\checkmark 1$ & $\checkmark 1$ & $\checkmark 1$ & $\checkmark 1$ \\
\hline Latam & 2014 & $\times 0$ & $\times 0$ & $\checkmark 1$ & $\times 0$ & $\checkmark 1$ & $\checkmark 1$ & $\checkmark 1$ & $\checkmark 1$ \\
\hline Avianca & 2015 & $\times 0$ & $\times 0$ & $\checkmark 1$ & $\times 0$ & $\checkmark 1$ & $\checkmark 1$ & $\checkmark 1$ & $\checkmark 1$ \\
\hline Latam & 2015 & $\times 0$ & $\times 0$ & $\checkmark 1$ & $\checkmark 1$ & $\checkmark 1$ & $\checkmark 1$ & $\checkmark 1$ & $\checkmark 1$ \\
\hline Avianca & 2016 & $\times 0$ & $\times 0$ & $\checkmark 1$ & $\checkmark 1$ & $\checkmark 1$ & $\checkmark 1$ & $\checkmark 1$ & $\checkmark 1$ \\
\hline Latam & 2016 & $\times 0$ & $\times 0$ & $\checkmark 1$ & $\checkmark 1$ & $\checkmark 1$ & $\checkmark 1$ & $\checkmark 1$ & $\checkmark 1$ \\
\hline Avianca & 2017 & $\times 0$ & $\checkmark 1$ & $\checkmark 1$ & $\checkmark 1$ & $\checkmark 1$ & $\checkmark 1$ & $\checkmark 1$ & $\checkmark 1$ \\
\hline Latam & 2017 & $\times 0$ & $\times 0$ & $\checkmark 1$ & $\checkmark 1$ & $\checkmark 1$ & $\checkmark 1$ & $\checkmark 1$ & $\checkmark 1$ \\
\hline Avianca & 2018 & $\times 0$ & $\times 0$ & $\checkmark 1$ & $\checkmark 1$ & $\checkmark 1$ & $\checkmark 1$ & $\checkmark 1$ & $\checkmark 1$ \\
\hline Latam & 2018 & $\times 0$ & $\times 0$ & $\checkmark 1$ & $\checkmark 1$ & $\checkmark 1$ & $\checkmark 1$ & $\checkmark 1$ & $\checkmark 1$ \\
\hline Viva Air & s.f. & $\times 0$ & $\times 0$ & $\times 0$ & $\times 0$ & $\times 0$ & $\times 0$ & $\times 0$ & $\times 0$ \\
\hline Avianca & 2019 & $\times 0$ & $\times 0$ & $\checkmark 1$ & $\checkmark 1$ & $\checkmark 1$ & $\checkmark 1$ & $\checkmark 1$ & $\checkmark 1$ \\
\hline Latam & 2019 & $\times 0$ & $\times 0$ & $\checkmark 1$ & $\times 0$ & $\checkmark 1$ & $\checkmark 1$ & $\times 0$ & $\checkmark 1$ \\
\hline Viva Air & s.f. & $\times 0$ & $\times 0$ & $\times 0$ & $\times 0$ & $\times 0$ & $\times 0$ & $\times 0$ & $\times 0$ \\
\hline
\end{tabular}

Fuente: Elaboración propia de los autores

En este sentido, Viva Air no presenta información relacionada con su interacción con Proveedores. No obstante, del análisis cualitativo se destaca que Avianca "...cuenta con una estructura de Gobierno Corporativo que le permite garantizar a sus grupos de interés una gestión administrativa recta y transparente, en cumplimiento del Pacto Social, las leyes y regulaciones que le son aplicables..." (2013, p. 13) y que gracias a su evolución ha construido el Código de Ética con el "...propósito de asegurar el cumplimiento de las disposiciones del Código de Ética y su adecuada interpretación, además de solucionar los conflictos de interés que se lleguen a presentar en el desarrollo de nuestras actividades..." (2019, p. 88).

Del mismo modo, LATAM se compromete a promover dentro de su gobierno corporativo el cumplimiento del “...desempeño y de los ejecutivos, mediante el Manual de manejo de información de Interés, el Código de ética para altos Ejecutivos, acuerdos de confidencialidad y el Formulario de Información Personas Relacionadas...” (2013, p. 26), esfuerzos que han permitido el desarrollo y 
Accountability, marketing relacional y grupos de interés en el transporte aéreo colombiano...

posicionamiento de la "...estructura de gobierno cuenta con cuatro comités que apoyan al Directorio con análisis y recomendaciones: Estrategia y Sostenibilidad, Liderazgo, Finanzas y Clientes...” (2019, p. 32).

\section{V.8 Factor 8 - sociedad}

La Tabla 8 resume el comportamiento sectorial relacionado con los aspectos evaluados dentro del factor de la "Sociedad", identificando así que el sector reconoce la importancia e impacto que tiene sobre las comunidades y para ello los agentes han desarrollado estrategias que responden al cumplimiento del Pacto Global, los Objetivos de Desarrollo Sostenible, la exigencia por la promoción de los derechos humanos y su papel en la inclusión y el desarrollo local.

De este modo, se evidencia que se ha dado un especial interés por responder a las expectativas de este stakeholder por medio de iniciativas filantrópicas, de inclusión social y en búsqueda de mejorar las condiciones de vida de las poblaciones vulnerables de las regiones donde presta su servicio.

En este sentido, Viva Air concentra sus acciones en fines netamente filantrópicos y donativos al "....realizar millones de sueños, lo enfocan en la calidad de vida y tanto en la prevención del cáncer de mama, uno de nuestros pilares desde que iniciamos operaciones..." (s/f). Adicionalmente, del análisis cualitativo, se destaca que Avianca desde 2012 "...cuenta con una serie de programas destinados a contribuir a la mejora de las condiciones de vida de poblaciones vulnerables y comunidades de su área de influencia...” (2013, p. 101) con el fin de hoy asumir su posición como "...adherentes al Pacto Global, nuestro compromiso con la agenda de Derechos Humanos y Empresa de las Naciones Unidas (ONU), los principios en materia de Trabajo Decente de la Organización Internacional del trabajo (OIT), la transparencia y la protección del ambiente...” (2019, p. 57).

Tabla 8. Observación del Factor 8 (Sociedad)

\begin{tabular}{|c|c|c|c|c|c|c|}
\hline \multirow[b]{2}{*}{ 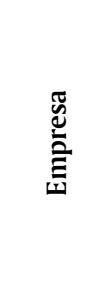 } & \multirow[b]{2}{*}{ Año } & \multicolumn{5}{|c|}{ Sociedad } \\
\hline & & 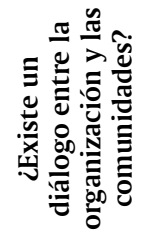 & 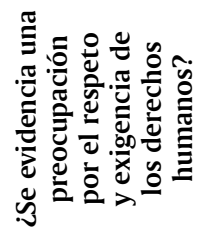 & 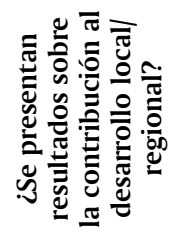 & 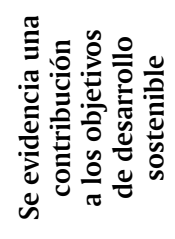 & 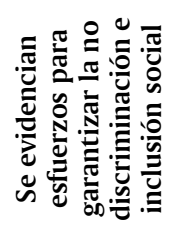 \\
\hline Avianca & 2012 & $\times 0$ & $\checkmark 1$ & $\begin{array}{ll} & 1\end{array}$ & $\times 0$ & $\times 0$ \\
\hline Latam & 2012 & $\times 0$ & $\checkmark 1$ & $\checkmark 1$ & $\checkmark 1$ & $\checkmark 1$ \\
\hline Avianca & 2013 & $\times 0$ & $\checkmark 1$ & $\checkmark 1$ & $\times 0$ & $\checkmark 1$ \\
\hline Latam & 2013 & $\times 0$ & $\checkmark 1$ & $\checkmark 1$ & $\checkmark 1$ & $\checkmark 1$ \\
\hline Avianca & 2014 & $\times 0$ & $\checkmark 1$ & $\checkmark 1$ & $\checkmark 1$ & $\times 0$ \\
\hline Latam & 2014 & $\times 0$ & $\checkmark 1$ & $\checkmark 1$ & $\checkmark 1$ & $\checkmark 1$ \\
\hline
\end{tabular}




\begin{tabular}{|c|c|c|c|c|c|c|}
\hline \multirow[b]{2}{*}{ 总 } & \multirow[b]{2}{*}{ Año } & \multicolumn{5}{|c|}{ Sociedad } \\
\hline & & 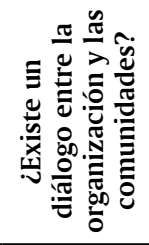 & 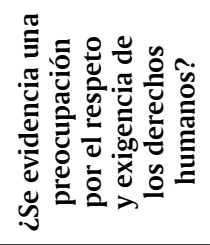 & 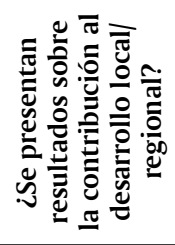 & 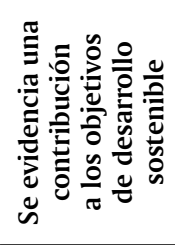 & 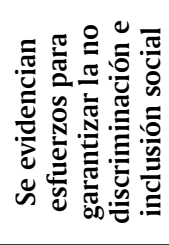 \\
\hline Avianca & 2015 & $\times 0$ & $\checkmark \quad 1$ & $\checkmark 1$ & $\times 0$ & $\checkmark 1$ \\
\hline Latam & 2015 & $\times 0$ & $\checkmark 1$ & $\checkmark 1$ & $\checkmark 1$ & $\checkmark 1$ \\
\hline Avianca & 2016 & $\times 0$ & $\checkmark 1$ & $\checkmark 1$ & $\checkmark 1$ & $\checkmark 1$ \\
\hline Latam & 2016 & $\times 0$ & $\checkmark 1$ & $\checkmark 1$ & $\checkmark 1$ & $\checkmark 1$ \\
\hline Avianca & 2017 & $\checkmark 1$ & $\checkmark 1$ & $\checkmark 1$ & $\checkmark 1$ & $\checkmark 1$ \\
\hline Latam & 2017 & $\times 0$ & $\checkmark 1$ & $\checkmark 1$ & $\checkmark 1$ & $\checkmark 1$ \\
\hline Avianca & 2018 & $\checkmark 1$ & $\checkmark 1$ & $\checkmark 1$ & $\checkmark 1$ & $\checkmark 1$ \\
\hline Latam & 2018 & $\times 0$ & $\checkmark 1$ & $\checkmark 1$ & $\checkmark 1$ & $\checkmark 1$ \\
\hline Viva Air & s.f. & $\times 0$ & $\times 0$ & $\checkmark 1$ & $\times 0$ & $\checkmark 1$ \\
\hline Avianca & 2019 & $\times 0$ & $\checkmark 1$ & $\checkmark 1$ & $\checkmark 1$ & $\checkmark 1$ \\
\hline Latam & 2019 & $\times 0$ & $\checkmark 1$ & $\checkmark 1$ & $\checkmark 1$ & $\checkmark 1$ \\
\hline Viva Air & s.f. & $\times 0$ & $\times 0$ & $\times 0$ & $\times 0$ & $\times 0$ \\
\hline
\end{tabular}

Fuente: Elaboración propia de los autores

Por otro lado, LATAM plantea desde 2012 que su relacionamiento no es una estrategia filantrópica, sino que responde a "...las necesidades y aspiraciones de la comunidad en general, en el corto, mediano y largo plazo...” (2013, p. 84), de forma que durante los últimos años ha fortalecido dicho programa mediante la agrupación de "...iniciativas locales dirigidas a la región para promover la conservación del medio ambiente y potenciar el turismo en un enfoque amplio, que considera la concientización sobre la sostenibilidad de las actividades turísticas..." (2019, p. 98).

\section{VI.Nivel de asociación de marketing relacional}

Finalmente, se calculó el Coeficiente de Concordancia W de Kendall con el fin de evaluar el nivel de asociación de marketing relacional sobre los ocho factores identificados dentro de las memorias de sostenibilidad. Este índice permite validar el nivel de acuerdo entre las unidades de observación, que para la presente investigación fueron las organizaciones. Así, se plantean las siguientes hipótesis:

H0: La concordancia de la rendición de cuentas de las organizaciones del sector de transporte aéreo colombiano se debe a las probabilidades. (Hipótesis nula)

H1: La concordancia de la rendición de cuentas de las organizaciones del sector de transporte aéreo colombiano no se debe a las probabilidades. (Hipótesis alternativa) 
Accountability, marketing relacional y grupos de interés en el transporte aéreo colombiano...

En este sentido, con el fin de mostrar la evaluación que se realizó al nivel de asociación de marketing relacional sobre cada uno de los factores; a continuación, se presenta el análisis que se realiza a la evaluación realizada al factor de los "Accionistas" dentro de las memorias de sostenibilidad. Así, al operar el Coeficiente de Concordancia W de Kendall se obtuvo como resultado un índice de 0.714 (o 71,4\%) de concordancia con una Chi-Cuadrado de 64.258 (6 g.l. y $\mathrm{P}=0.000$ ), conforme a la Tabla 9 .

Por lo tanto, a partir de los ítems verificados en la matriz de observación y registro para este factor, se rechaza la hipótesis nula indicando así que hay concordancia estadísticamente significativa en relación con la metodología de rendición de cuentas de las organizaciones que componen el sector, de cara al grupo de "Accionistas".

Tabla 9. Estadísticos de prueba Factor 2 (Accionistas)

\begin{tabular}{ll}
\hline $\mathrm{N}$ & 15 \\
$\mathrm{~W}$ de Kendall & \\
Chi-cuadrado & 0,714 \\
$\mathrm{gl}$ & 64,258 \\
Sig. asintótica & 0,000 \\
\hline
\end{tabular}

a. Coeficiente de concordancia de Kendall

Fuente: elaboración propia a partir del paquete estadístico SPSS

En secuencia a lo anterior, para cada uno de los factores se obtuvieron como índices los presentados en la Tabla 10, los cuales implican el rechazo de las hipótesis nulas para cada uno. Esto indica que, a partir de los ítems verificados en las matrices de observación y registro correspondientes, hay concordancia estadísticamente significativa en relación con la metodología de rendición de cuentas del sector frente a los ocho grupos de interés o factores.

Tabla 10. Estadísticos de prueba Factor 8 (Sociedad)

\begin{tabular}{lcccc}
\hline \multicolumn{1}{c}{ Factor } & Coeficiente W de Kendall & Chi-Cuadrado & P-Value & Se rechaza Ho \\
\hline Factor 2 (Accionistas) & 0,675 & 72,904 & 0,000 & $\mathrm{SI}$ \\
Factor 6 (Proveedores) & 0,620 & 55,758 & 0,000 & $\mathrm{SI}$ \\
Factor 5 (Medioambiente) & 0,618 & 89,038 & 0,000 & $\mathrm{SI}$ \\
Factor 7 (Gobernanza) & 0,601 & 75,746 & 0,000 & $\mathrm{SI}$ \\
Factor 8 (Sociedad) & 0,592 & 42,629 & 0,000 & $\mathrm{SI}$ \\
Factor 4 (Consumidores) & 0,356 & 44,800 & 0,000 & $\mathrm{SI}$ \\
Factor 3 (Colaboradores) & 0,537 & 67,638 & 0,000 & $\mathrm{SI}$ \\
Factor 1 (Información) & 0,171 & 24,582 & 0,002 & $\mathrm{SI}$ \\
\hline
\end{tabular}

Fuente: elaboración propia a partir del paquete estadístico SPSS 


\section{Discusiones}

El sector aéreo es uno de los más relevantes para la política pública nacional en relación con su potencial de crecimiento y el impacto que tiene en la economía. Al centrar el análisis en el transporte aéreo colombiano, se identificó que existe una posición dominante de las empresas Avianca y LATAM; de modo que se corrobora la premisa de Cowper-Smith \& de Grosbois (2011) en relación con que las investigaciones relacionadas con este sector son escasas, por las condiciones oligopólicas propias del mercado.

Así pues, el principal aporte de esta investigación se centra en la contribución en la provisión de acercamientos al conocimiento especializado sobre el proceso de accountability en el sector de transporte aéreo como herramienta de marketing relacional, a partir de la experiencia evidenciada en el ámbito colombiano.

Ahora bien, conforme a lo planteado por Hinson (2011) y Karaman \& Akman (2018), la evolución de las organizaciones, y en especial las del sector de transporte aéreo en Colombia, presentan una rápida curva de aprendizaje sobre las expectativas de sus grupos de interés, los estándares internacionales y el nivel de detalle requerido por los mismos a la hora de rendir cuentas. De modo que incrementar la información sobre indicadores, iniciativas, proyecciones y aspectos relevantes, han perfeccionado los requerimientos mínimos de buenas prácticas de accountability, al pasar de un número relativamente pequeño de aspectos materiales a divulgar. Lo anterior, Rezaee (2017) los planteó como el crecimiento vertiginoso de la información de carácter no financiero, buscando satisfacer a los stakeholders y mantener una relación estrecha con los mismos (Kuo et al., 2016).

Es de resaltar que aun cuando el sector aeronáutico no conlleva un efecto negativo sobre la sociedad, se evidencian aspectos materiales fundamentales a la hora de rendir cuentas con sus grupos de interés. De modo que, la recomendación presentada por Da Silva et al. (2015) ha tomado lugar en la gestion interna de las organizaciones en pro depresentar informacion detallada de los impactos del objeto social. De esta forma, en el contexto colombiano se encuentra una alta disposición a gestionar la relación con el medioambiente, confirmando así lo que Robinson (2015) manifestó como el interés por reducir las emisiones de carbono, los residuos e impactos comunitarios.

Esta situación se interrelaciona con el eje de Gobernanza, puesto que, en las organizaciones colombianas, los comités medioambientales no han tomado un papel estratégico en la toma de decisiones del gobierno corporativo, a diferencia de lo que se plantea ampliamente la literatura académica (Becerra Velasco, Espinosa Ávila, Guzmán Hurtado, \& Rodríguez Vargas, 2017; Jouini, Ajina, \& Derbali, 2018; Thompson, Alleyne, \& Charles-Soverall, 2019). 
Accountability, marketing relacional y grupos de interés en el transporte aéreo colombiano...

En este sentido, de acuerdo con Cowper-Smith \& de Grosbois (2011), se evidencia una marcada preocupación por mantener un contacto cercano y duradero con la sociedad y el compromiso con los colaboradores (bienestar y equidad), pues pueden ser considerados como ejes centrales de las partes interesadas primarias (Gupta \& Agrawal, 2016; Kim \& Kim, 2019).

No obstante, al evaluar la propuesta de Franklin \& Oehmke (2019) y de Salmi $\&$ Khan (2019), las organizaciones colombianas del sector de transporte aéreo colombiano aún no han implementado iniciativas de relacionamiento enfocadas al establecimiento de diálogos y compromisos conjuntos con los grupos de interés dentro de sus informes. Esta falencia puede conllevar a perder de vista los asuntos materiales y su constante evolución.

En ese sentido, la mirada académica y el análisis observacional sobre los procesos de accountability al interior de las prácticas empresariales del sector, se concluye que la entrada en vigor de la Directriz Europea generó una expectativa en estas organizaciones de tal forma que a partir de 2017 se evidencia una preocupación por aumentar la información divulgada en relación con cada uno de los stakeholders y certificar dichos documentos a través de verificadores externos.

Por otro lado, los datos obtenidos permiten concluir que el sector empresarial de transporte aéreo colombiano se puede categorizar en dos subgrupos; el primero, compuesto por Avianca y LATAM, quienes han desarrollado estrategias de marketing relacional en su proceso de rendición de cuentas, siguiendo las recomendaciones que plantean Kim \& Kim (2019) y Souza-Monteiro \& Hooker (2017); mientras que el segundo, compuesto por Viva Air y los demás agentes del mercado, buscan que sus interacciones con los grupos de interés sea a través de diferentes comunicaciones y múltiples sitios web, de modo que no se centraliza la información.

Por otra parte, una vez contrastadas las investigaciones previas y caracterizado el comportamiento de las organizaciones del sector, se hace necesario evaluar el comportamiento de accountability del sector de transporte aéreo en Colombia en conjunto; para ello la Tabla 10 resume los resultados obtenidos en los Coeficientes de Concordancia W de Kendall, presentando que si bien todos los grupos hacen parte de la rendición de cuentas, la relación con ciertos grupos cuenta con un mayor nivel de gestión de mercado.

Así bien, se evidencia que los principales grupos de interés a los que se dirige el proceso de rendición de cuentas dentro del sector son a los Accionistas, Proveedores e interesados en la estructura de Gobernanza Corporativa, comportamiento comúnmente evidenciado en cualquier industria y que se espera dado el flujo de caja que genera el sector. Así mismo, los aspectos relacionados con su impacto Social, el Cliente y la disponibilidad de la Información son los aspectos de menor congruencia en el agregado, lo que indica que hay una disparidad en la metodología de rendición de cuentas entre las 
organizaciones; sin embargo, se debe destacar que en conjunto el sector realiza el proceso de accountability de forma congruente.

En relación con las limitaciones, al no disponer de información ampliamente compartida por Viva Colombia y las demás organizaciones (no dominantes en el mercado), la evaluación del comportamiento de rendición de cuentas dentro del sector de transporte aéreo colombiano queda sujeto a las iniciativas de Avianca y LATAM; que, si bien concentran cerca del $76 \%$ del mercado, aún queda un $25 \%$ restante que no se pudo observar metodológicamente y resulta relevante investigar.

No obstante, los aportes que plantea este estudio se centran en evidenciar que el proceso de accountability no solo debe ser visto como un requisito de información que normativamente se prevé entre en vigor, sino que representa una oportunidad para que las organizaciones gestionen su relación con los grupos de interés y generen estrategias para alinear sus esfuerzos en la búsqueda por posicionarse en el mercado.

\section{Conclusiones}

El sector de transporte aéreo en Colombia ha sido uno de los más impactados por la pandemia de la COVID-19 y al ser un sector que facilita la conexión de bienes y personas, resulta clave para la recuperación económica del país. Este se encuentra compuesto principalmente por Avianca, LATAM y Viva Colombia, organizaciones que sistemáticamente pueden ser vistas como sistemas abiertos que interactúan con sus grupos de interés para gestionar procesos que permitan crear valor dentro de la organización, el cual se distribuye entre todos los stakeholders.

En ese sentido, la contabilidad en las organizaciones cumple un papel mediador del conflicto que se genera entre los distintos integrantes del contrato organizacional, evitando así la desintegración de estas gracias conocimiento común sobre el grado de cumplimiento de los acuerdos; reflexión que en otras palabras se resume en facilitar la relación de accountability entre las partes interesadas. Es por esto que la divulgación de información no financiera ha tomado gran importancia a nivel internacional, y más aún cuando revela detalles que fortalecen la relación entre cada grupo de interés y la organización.

Así bien, los informes de gestión, memorias de sostenibilidad o reportes integrados han sido una de las principales estrategias de comunicación de este tipo de información no financiera, de modo que a menudo terminan conllevando consigo un enfoque de marketing relacional, con el fin de cumplir con las expectativas de sus grupos de interés y gestionar su interacción desde el interior de la organización.

De esta forma, la presente investigación se propuso evaluar la práctica del accountability como herramienta de marketing relacional a los grupos de interés dentro del sector del transporte aéreo colombiano, por medio de la contrastación de premisas identificadas en el proceso de revisión sistemática 
Accountability, marketing relacional y grupos de interés en el transporte aéreo colombiano...

de literatura y la caracterización del comportamiento del sector empresarial colombiano, a partir del desarrollo de una matriz de evaluación y concordancia.

A partir de lo anterior, se realizó la revisión sistemática de literatura e identificaron las premisas que permitieron la construcción de los ítems de marketing relacional a evaluar dentro de la matriz de observación y registro. De esta forma, se caracterizó el grado de relacionamiento y el comportamiento de accountability de las principales organizaciones frente a las expectativas registradas por la literatura y los factores validados con los jueces que revisaron la matriz.

Así pues, el ejercicio evidenció que hay un alto nivel de divulgación de información no financiera en el sector de transporte aéreo colombiano, generando mayores niveles de confianza y relación a largo plazo con los grupos de interés. Este efecto es más constante, en la medida que estas organizaciones buscan que la información presentada se encuentre auditada por una firma que de mayor confiabilidad.

En relación con los grupos de interés, uno de los principales ejes es el Accionista, quien busca conocer elementos cualitativos de la compañía y su capacidad para continuidad en el futuro. Así mismo, el transporte aéreo al ser un servicio donde el contacto con los colaboradores es fundamental, las organizaciones del sector mantienen una relación estrecha y cordial con este grupo de interés a través de programas e iniciativas que hacen atractivo su papel como empleador.

Los proveedores presentan una relación antagonista, toda vez que, al ser industrias especializadas y centralizadas en oligopolios tecnológicos, no evidencian un gran impacto en la pequeña industria local. No obstante, una de las partes interesadas más importantes para la industria será el medioambiente, pues hay un fuerte interés operativo y técnico en medir el impacto de su operación, aun cuando estratégicamente se requiere un mayor interés institucional para fortalecer este aspecto desde la gobernanza corporativa.

Por otro lado, y no menos importante, el acercamiento manifestó que las organizaciones del sector están centradas en generar códigos de ética y de gobernanza; sin embargo, se evidencia la necesidad de fortalecer la política organizacional en contra del lavado de activos y financiación del terrorismo como asunto material de la sociedad y del gobierno.

Esta aproximación se aplicó sobre la información publicada oficialmente por estas organizaciones, la cual se recopiló para posteriormente ser analizada mediante estadística no paramétrica, obteniendo así que el comportamiento del sector en conjunto es concordante entre las diferentes organizaciones y para los ocho factores analizados. En este resultado, se evidencia claramente una mayor revelación consistente en relación con los Accionistas o Inversores, mientras que el cumplimiento de las expectativas sociales y del consumidor aún requieren un mayor compromiso con el proceso de divulgación de la información. 
De este modo, la presente investigación proporciona una perspectiva diferencial que analiza el comportamiento de accountability como herramienta de marketing relacional dirigido a los grupos de interés dentro del sector de transporte aéreo colombiano, abriendo un espacio que permite la interdisciplinariedad entre el dialogo contable y de marketing en el campo académico. Así como, la búsqueda Gerencial en el sector, por gestionar la relación con sus stakeholders a través de la alineación de su estrategia de rendición de cuentas y de marketing relacional. Además, resultado de esta investigación se presenta una matriz que evalúa ocho factores relacionales con sus respectivos ítems para futuras investigaciones ya sea en el contexto nacional o internacional.

En contraste, una de las limitaciones presentes no sólo en este estudio, sino en los que basan su análisis en la información que es verificable a través de fuentes públicas abiertas, es que no es posible profundizar en el análisis de las cifras, datos, hechos, según las categorías y variables que se trazan en el problema de investigación a partir del uso de los datos directos de la organización, más allá de los que en ellas se procesan y divulgan. Lo anterior, abre una oportunidad a invitar a las organizaciones o grupos de ellas a participar en estudios de caso que deriven en un acceso a los datos con menores restricciones.

Adicionalmente, otra reflexión que surge a partir del estudio es el abordaje para conceptualizar la relación entre las variables accountability y conexión con los grupos de interés, toda vez que, si bien en este documento se realiza desde el marketing relacional desde su noción holística, otra vertiente válida son las comunicaciones integradas de marketing, las relaciones públicas y, en el marco de ellas, el manejo de la reputación e imagen institucional.

Finalmente, se recomienda que las futuras investigaciones propendan por realizar comparaciones integrales de los ocho factores propuestos, contrastando hermenéuticamente el nivel de transparencia de la rendición de cuentas oficial emitida por el gobierno corporativo y la socializada mediante fuentes secundarias de información; así mismo, evaluar la percepción del proceso de accountability directamente con los grupos de interés, de modo que se determine si la imagen emitida en los documentos de información no financiera es percibida bajo la misma línea en que se desarrolló, cumpliendo así con el objetivo de gestionar las relaciones con los stakeholders.

\section{Referencias bibliográficas}

Aeronáutica Civil. (2021). Entre septiembre de 2020 y enero de 2021 más de 6 millones de personas viajaron por avión en medio de la reactivación del transporte aéreo en el país. Recuperado de https:/www.aerocivil.gov.co/prensa/noticias/Pages/Entreseptiembre-de-2020-y-enero-de-2021-mas--de-6-millones-de-personas-viajaron-poravion-en-medio-de-la-reactivacion.aspx 
Accountability, marketing relacional y grupos de interés en el transporte aéreo colombiano...

Airports Council International. (2017). Annual World Airport Traffic Forecasts 2017-2040. Recuperado de https:/aci.aero/wp-content/uploads/2019/10/WATF_2017.pdf

Andriof, J., \& McIntosh, M. (2017). Perspectives on corporate citizenship. En Perspectives on Corporate Citizenship. https://doi.org/10.4324/9781351282369

Avianca. (2020b). Proyecto de no distribución de utilidades.

Avianca. (2020a). Informe Anual (2019).

Avianca. (2019). Informe Anual (2018).

Avianca. (2018). Informe Anual (2017).

Avianca. (2017). Informe de Gestión (2016).

Avianca. (2016). Informe de Responsabilidad Social \& Sostenibilidad (2015).

Avianca. (2015). Informe de Responsabilidad Social \& Sostenibilidad (2014).

Avianca. (2014). Informe de Responsabilidad Social \& Sostenibilidad (2013).

Avianca. (2013). Informe de Responsabilidad Social \& Sostenibilidad (2012).

Babor, T. F., Robaina, K., \& Jernigan, D. (2015). The influence of industry actions on the availability of alcoholic beverages in the African region. Addiction, 110(4), 561-571. https://doi.org/10.1111/add.12832

Bachman, B. J., Bashyal, S., \& Baumann, M. (2012). Sustainability in the plastics industry: Concerns, issues, and strategies. Polimeri, 33(1), 6-11.

Barrera Cifuentes, J. P., \& Gutiérrez Roa, R. D. (2015). Rendición de cuentas on-line de los partidos y movimientos políticos en Colombia. Universidad de La Salle.

Batista, N. (2018). Integrated reporting stimulates strategic communication of corporate social responsibility? A marketing perspective analysis based on Maignan, Ferrell and Ferrell's stakeholder model of corporate social responsibility in marketing. Media and Jornalismo, 18(33), 43-59. https://doi.org/10.14195/2183-5462_33_3

Becerra Velasco, C. J., Espinosa Ávila, E., Guzmán Hurtado, M., \& Rodríguez Vargas, P. (2017). Impacto de las buenas prácticas de gobierno corporativo en la generación de valor financiero de las empresas del sector de transporte aéreo en Colombia. Recuperado de http://tesis.pucp.edu.pe/repositorio/handle/20.500.12404/9011

Belanche, D., Casaló, L. V, Flavián, C., \& Schepers, J. (2020). Service robot implementation: a theoretical framework and research agenda. The Service Industries Journal, 40(34), 203-225. https://doi.org/10.1080/02642069.2019.1672666

Beltrán, Ó. (2005). Revisiones sistemáticas de la literatura. Revista Colombiana de Gastroenterología, 20(1), 60-69.

Católico, D. F. (2011). Revelación y divulgación de la información financiera y no financiera de las universidades públicas en Colombia. Revista Facultad de Ciencias Económicas: Investigación y Reflexión, 20(1), 57-76. https://doi.org/https://doi.org/10.18359/ rfce. 2185

Católico-Segura, D. F., Gómez-Díaz, A. J., \& Gómez-Solano, P. A. (2014). Revelación on-line de la información financiera y no financiera de las entidades de la rama ejecutiva del orden. Cuadernos de Contabilidad, 15(37), 77-107. https://doi.org/10.11144/ Javeriana.cc15-37.roif 
Chicaiza-Becerra, L., Riaño-Casallas, M. I., Rojas-Berrio, S. P., \& Garzón-Santos, C. (2017). Revisión Sistemática de la Literatura en Administración (Núm. 29). https://doi. org/10.13140/RG.2.2.15345.89443

Clavijo, S. (2018). Sector transporte: desempeño 2017 y perspectivas 2018. La República. Recuperado de https://www.larepublica.co/analisis/sergio-clavijo-500041/sectortransporte-desempeno-2017-y-perspectivas-2018-2743055

Cowper-Smith, A., \& de Grosbois, D. (2011). The adoption of corporate social responsibility practices in the airline industry. Journal of Sustainable Tourism, 19(1), 59-77. https:// doi.org/10.1080/09669582.2010.498918

Crocker, R. (2014). 'Ethicalization' and greenwashing: Business, sustainability and design. En Design for Business.

Da Silva, R. C. F., Seibert, R. M., Wbatuba, B. B. R., \& Macagnan, C. B. (2015). The good practices of governance: A study on the transparency and accountability in the Oscips - RS | As boas práticas de governança: Um estud o sobre a transparência e prestação de contas nas Oscips do RS. Revista de Gestao Social e Ambiental, 9(3), 24-44.

De Gortari, E. (1968). Lógica General. La impresora azteca S. de R. L.

Deegan, C. (2014). Financial Accounting Theory (McGraw-Hill Education, Ed.). Australia.

Dumitru, M., Guşe, R. G., Feleagă, L., Mangiuc, D. M. D. M., Feldioreanu, A. I. A. I., Gușe, R. G., ... Feldioreanu, A. I. A. I. (2015). Marketing communications of value creation in sustainable organizations. The practice of integrated reports. Amfiteatru Economic, 17(40), 955-976.

Dumitru, M., Gușe, R. G., Feleagă, L., Mangiuc, D. M., \& Feldioreanu, A. I. (2015). Marketing Communications of Value Creation in Sustainable Organizations. The Practice of Integrated Reports. Amfiteatru Economic, 17(40), 955-976.

Dwyer, R. J. (2007). Alleviating poverty: How do we know the scope of the problem and when we have solved it? Management Decision, 45(8), 1344-1358. https://doi. org/10.1108/00251740710819078

Ernst \& Young. (2018). Novedades sobre información no financiera y diversidad.

Espinosa-Flórez, G. E. (2011). Los servicios de revisión: una alternativa para el aseguramiento de información financiera. Cuadernos de Contabilidad, Vol. 12, pp. 327-352. Pontificia Universidad Javeriana, Facultad de Ciencias Económicas y Administrativas, Departamento de Ciencias Contables.

Franklin, K., \& Oehmke, J. (2019). Building African Agribusiness through Trust and Accountability. Journal of Agribusiness in Developing and Emerging Economies, 9(1), 22-43. https://doi.org/10.1108/JADEE-01-2018-0005

Freeman, R. E. (1984). Strategic management: A stakeholder approach (Pitman Pub). Boston, United States of America.

Freeman, R. E., Harrison, J. S., Wicks, A. C., Parmar, B., \& de Colle, S. (2010). Stakeholder theory: The state of the art. En Stakeholder Theory: The State of the Art. https://oi. org/10.1017/СBO9780511815768

Gómez-Meneses, F. E., \& Católico-Segura, D. F. (2009). Revelación y divulgación de la información financiera y no financiera on-line de las 500 empresas más representativas en Colombia*. Cuadernos de Contabilidad, 10(27), 269-318. 
Accountability, marketing relacional y grupos de interés en el transporte aéreo colombiano...

Groninger, K. (2010). Museum accountability in America: How public expectations stimulate fiscal and ethical transparency. International Journal of the Inclusive Museum, 2(4), 125-136. https://doi.org/10.18848/1835-2014/CGP/v02i04/44284

Gupta, R., \& Agrawal, G. (2016). Corporate social responsibility in emerging economies: Assessing environmental reporting of Indian firms. International Business Management, 10(9), 1575-1580. https://doi.org/10.3923/ibm.2016.1575.1580

Hinson, R. (2011). Corporate social responsibility (CSR) reportage on websites and in annual reports: The case of CAL Bank in Ghana. Corporate Ownership and Control, 8(2 D), 190-202. https://doi.org/10.22495/cocv8i2c1p4

Irvine, K., \& O’Brien, S. (2009). Progress on stakeholder participation in the implementation of the water framework directive in the republic of Ireland. Biology and Environment, 109(3), 365-376. https://doi.org/10.3318/BIOE.2009.109.3.365

Jernigan, D. H., \& Babor, T. F. (2015). The concentration of the global alcohol industry and its penetration in the African region. Addiction, 110(4), 551-560. https://doi. org/10.1111/add.12468

Jouini, F., Ajina, A., \& Derbali, A. (2018). Corporate governance and corporate social responsibility. International Journal of Management and Enterprise Development, 17(2), 155-167. https://doi.org/10.1504/JJMED.2018.090841

Karaman, A. S., \& Akman, E. (2018). Taking-off corporate social responsibility programs: An AHP application in airline industry. Journal of Air Transport Management, 68, 187197. https://doi.org/10.1016/J.JAIRTRAMAN.2017.06.012

Kim, M., \& Kim, Y. (2019). CSR and Shareholder Value in the Restaurant Industry: The Roles of CSR Communication Through Annual Reports. Cornell Hospitality Quarterly, 60(1), 69-76. https://doi.org/10.1177/1938965518777969

Kitchenham, B. (2004). Procedures for Performing Systematic Reviews.

Kitchenham, B., Pretorius, R., Budgen, D., Pearl Brereton, O., Turner, M., Niazi, M., \& Linkman, S. (2010). Systematic literature reviews in software engineering - A tertiary study. Information and Software Technology, 52(8), 792-805. https://doi.org/https:// doi.org/10.1016/j.infsof.2010.03.006

Kuo, T. C., Kremer, G. E. O., Phuong, N. T., \& Hsu, C.-W. (2016). Motivations and barriers for corporate social responsibility reporting: Evidence from the airline industry. Journal of Air Transport Management, 57, 184-195. https://doi.org/10.1016/J. JAIRTRAMAN.2016.08.003

LATAM. (2020). Memoria Integrada Anual (2019).

LATAM. (2019). Memoria Integrada (2018).

LATAM. (2018). Reporte de Sostenibilidad (2017).

LATAM. (2017). Reporte de Sostenibilidad (2016).

LATAM. (2016). Reporte de Sostenibilidad (2015).

LATAM. (2015). Reporte de Sostenibilidad (2014).

LATAM. (2014). Reporte de Sostenibilidad (2013).

LATAM. (2013). Reporte de Sostenibilidad (2012). 
Lee, S., Seo, K., \& Sharma, A. (2013). Corporate social responsibility and firm performance in the airline industry: The moderating role of oil prices. Tourism Management, 38, 20-30. https://doi.org/10.1016/J.TOURMAN.2013.02.002

Luo, J. M., \& Lam, C. F. (2016). Corporate social responsibility and responsible gambling in a gaming destination. En Corporate Social Responsibility and Responsible Gambling in Gaming Destinations.

Luo, J. M., Lam, C. F., Chau, K. Y., Shen, H. W., \& Wang, X. (2017). Measuring corporate social responsibility in gambling industry: Multi-items stakeholder based scales. Sustainability (Switzerland), 9(11). https://doi.org/10.3390/su9112012

Mahmud, R., Manan, N. S. A., Hashim, M., Nor, N. H. M., \& Azizan, S. S. (2017). The use of social media for corporate reporting. Advanced Science Letters, 23(8), 7391-7393. https://doi.org/10.1166/asl.2017.9482

Mayoral, J., \& Segura, A. (2008). Gobierno corporativo, conflictos de agencia y elección de auditor / Corporate Governance, agency conflicts and auditor choice. Revista Española de Financiación y Contabilidad, 37(137), 113-135.

Nelson, J. (2014). Corporate social responsibility: Emerging good practice for a New Era. OECD Observer, (299), 9-10.

O'Malley, L. (2014). Relational marketing: development, debates and directions, Journal of Marketing Management, 30(11-12), 1220-1238, https://doi.org/10.1080/026725 7X.2014.939592

Parlamento Europeo, \& Consejo de la Unión Europea. (2014). Directiva 2014/95/UE. Estrasburgo.

Paul, J. (2019). Marketing in emerging markets: a review, theoretical synthesis, and extension. International Journal of Emerging Markets, 15(3), 446-468. https://doi. org/10.1108/JOEM-04-2017-0130

Paul, J., \& Benito, G. R. G. (2018). A review of research on outward foreign direct investment from emerging countries, including China: what do we know, how do we know and where should we be heading? Asia Pacific Business Review, 24(1), 90-115. https://oi. org/10.1080/13602381.2017.1357316

Pedersen, E. R. G., \& Andersen, K. R. (2015). Sustainability innovators and anchor draggers: A global expert study on sustainable fashion. Journal of Fashion Marketing and Management, 19(3), 315-327. https://doi.org/10.1108/JFMM-08-2014-0059

Pérez-Rave, J., Álvarez-Jaramillo, G., \& Henao-Velásquez, J. (2012). ¿ Cómo identificar la literatura "poco - vital" y cuantificar su representatividad ? Aplicación al problema del vendedor viajero $<$ TSP $>$. Ingeniería Industrial: Actualidad y Nuevas Tendencias, $8,51-60$.

Portafolio. (2019). Transporte aéreo cerró el 2018 con cifras récord. Recuperado el 29 de octubre de 2019, de https:/www.portafolio.co/economia/transporte-aereo-cerro-el2018-con-cifras-record-525394

Power, M. K. (2007). Corporate governance, reputation, and environmental risk. Environment and Planning C: Government and Policy, 25(1), 90-97. https://oi. org/10.1068/c0619j 
Accountability, marketing relacional y grupos de interés en el transporte aéreo colombiano...

Rana, J., \& Paul, J. (2017). Consumer behavior and purchase intention for organic food: A review and research agenda. Journal of Retailing and Consumer Services, 38, 157165. https://doi.org/10.1016/j.jretconser.2017.06.004

Rashid, M., Abdeljawad, I., Ngalim, S. M., \& Hassan, M. K. (2013). Customer-centric corporate social responsibility: A framework for Islamic banks on ethical efficiency. Management Research Review, 36(4), 359-378. https://doi.org/10.1108/01409171311314978

Rezaee, Z. (2017). Business sustainability: Performance, compliance, accountability and integrated reporting. En Business Sustainability: Performance, Compliance, Accountability and Integrated Reporting. https://doi.org/10.4324/9781351284288

Robinson, M. (2015). Completing (extending) the story on airline operational performance... advanced aviation weather analytics for improved air traffic impact awareness. AGIFORS 55th Annual Symposium: Analytics for Efficiency and Customer Centric Optimization.

Rodríguez de Ramírez, M. del C. (2012). Revisitando el concepto de accountability. En Universidad de Buenos Aires (Ed.), Contabilidad, Accountability y Transparencia: identificando tendencias (p. 230). Facultad de Ciencias Económicas.

Romolini, A., Fissi, S., \& Gori, E. (2014). Scoring CSR reporting in listed companies - evidence from Italian best practices. Corporate Social Responsibility and Environmental Management, 21(2), 65-81. https://doi.org/10.1002/csr.1299

Ross, S. (1973). The Economic Theory of Agency: The Principalıs Problem. The American Economic Review, 63(2), 134-139.

Salmi, H. A., \& Khan, F. R. (2019). A comparative case study on accountability of corporate social responsibility (csr) practices in oman lng and omifco at sur city in oman. Humanities and Social Sciences Reviews, 7(5), 490-502. https://doi.org/10.18510/ hssr.2019.7556

Saunders, M., Lewis, P., \& Thornhill, A. (2016). Research Methods For Business Students (Séptima). Londres: Pearson Education Limited.

Semana. (2019). El imparable ascenso del sector aéreo colombiano. Recuperado de https:// www.semana.com/contenidos-editoriales/el-tiempo-vuela/articulo/crecimiento-delsector-aereo-en-colombia/616592

Serry, N. M. (2019). Agile model for measurement of stockholders' performance in EPC company's in the context of sustainability. Offshore Mediterranean Conference and Exhibition 2019, OMC 2019.

Silvestri, A., Veltri, S., Venturelli, A., \& Petruzzelli, S. (2017). A research template to evaluate the degree of accountability of integrated reporting: A case study. Meditari Accountancy Research, 25(4), 675-704. https://doi.org/10.1108/MEDAR-11-2016-0098

Singh, R., \& Agarwal, S. (2013). Does CSR orientation reflect stakeholder relationship marketingorientation?Anempirical examination of Indian banks. Marketing Intelligence and Planning, 31(4), 405-420. https://doi.org/10.1108/02634501311324870

Souza-Monteiro, D., \& Hooker, N. (2017). Comparing UK food retailers corporate social responsibility strategies. British Food Journal, 119(3), 658-675. https://doi. org/10.1108/BFJ-04-2016-0152 
Sunder, S. (2005). La contabilidad y el modelo contractual de la empresa. En Universidad Nacional de Colombia (Ed.), Teoría de la contabilidad y el control (p. 334). Facultad de Ciencias Económicas, Doctorado en Ciencias Económicas.

Sweeney, L., \& Coughlan, J. (2008). Do different industries report Corporate Social Responsibility differently? An investigation through the lens of stakeholder theory. Journal of Marketing Communications, 14(2), 113-124. https://doi. org/10.1080/13527260701856657

Thompson, R. M., Alleyne, P., \& Charles-Soverall, W. (2019). Exploring governance issues among boards of directors within state-owned enterprises in Barbados. International Journal of Public Sector Management, 32(3), 264-281. https://oi.org/10.1108/ IJPSM-04-2018-0099

Tripathi, N., Seppänen, P., Boominathan, G., Oivo, M., \& Liukkunen, K. (2019). Insights into startup ecosystems through exploration of multi-vocal literature. Information and Software Technology, 105, 56-77. https://doi.org/10.1016/j.infsof.2018.08.005

Unidad Administrativa Especial de Aeronáutica Civil. (2018). Nota de Estudio - Foro Plan Estratégico Aeronáutico 2018-2030.

Viva Air. (s/f). Responsabilidad social Viva Air - Descubre más de nosotros. Recuperado el 25 de noviembre de 2019, de https:/www.vivaair.com/co/es/corporativo/responsabilidad-social

Waller, D. S., \& Lanis, R. (2009). Corporate social responsibility (CSR) disclosure of advertising agencies: An exploratory analysis of six holding companies annual reports. Journal of Advertising, 38(1), 109-121. https://doi.org/10.2753/JOA0091-3367380107

Wickham, M., Lehman, K., \& French, L. (2015). Communicating marketing priorities in the not-for-profit sector: A content analysis of australian state-museums' annual reports. Corporate Ownership and Control, 12(2Continued), 318-328.

Williamson, O. E. (1989). Las Instituciones económicas del capitalismo. México, D.F.: Fondo de Cultura Económica.

Williamson, O. E. (1996). The Mechanisms of Governance. Oxford, New York: Oxford University Press. 